\title{
The Neoepitopes on Methylglyoxal- (MG-) Glycated Fibrinogen Generate Autoimmune Response: Its Role in Diabetes, Atherosclerosis, and Diabetic Atherosclerosis Subjects
}

\author{
Shahnawaz Rehman $\mathbb{D}^{1},{ }^{1}$ Jiantao Song, ${ }^{2}$ Mohammad Faisal $\mathbb{D}^{,},{ }^{3}$ Abdulrahman A. Alatar, ${ }^{3}$ \\ Firoz Akhter $\left({ }^{4},{ }^{4}\right.$ Saheem Ahmad $\oplus,{ }^{5}$ and Bo $\mathrm{Hu}\left(\oplus^{2}\right.$ \\ ${ }^{1}$ Department of Biochemistry, Sir Syed Faculty of Science, Mohammad Ali Jauhar University, Rampur, U.P., India \\ ${ }^{2}$ Department of Emergency, Shandong Provincial Hospital Affiliated to Shandong First Medical University, Jinan, \\ Shandong 250021, China \\ ${ }^{3}$ Department of Botany \& Microbiology, College of Science, King Saud University, P.O. Box 2455, Riyadh 11451, Saudi Arabia \\ ${ }^{4}$ Department of Biomedical Engineering, Stony Brook University, New York, USA \\ ${ }^{5}$ Department of Medical Laboratory Sciences, College of Applied Medical Sciences, University of Hail, 2440, Saudi Arabia
}

Correspondence should be addressed to Bo Hu; hubo200015@sina.com

Received 21 December 2020; Revised 15 November 2021; Accepted 17 November 2021; Published 21 December 2021

Academic Editor: Jayeeta Ghose

Copyright (c) 2021 Shahnawaz Rehman et al. This is an open access article distributed under the Creative Commons Attribution License, which permits unrestricted use, distribution, and reproduction in any medium, provided the original work is properly cited.

\begin{abstract}
Objectives. In diabetes mellitus, hyperglycemia-mediated nonenzymatic glycosylation of fibrinogen protein plays a crucial role in the pathogenesis of micro- and macrovascular complications especially atherosclerosis via the generation of advanced glycation end products (AGEs). Methylglyoxal (MG) induces glycation of fibrinogen, resulting in structural alterations that lead to autoimmune response via the generation of neoepitopes on protein molecules. The present study was designed to probe the prevalence of autoantibodies against MG-glycated fibrinogen (MG-Fib) in type 2 diabetes mellitus (T2DM), atherosclerosis (ATH), and diabetic atherosclerosis (T2DM-ATH) patients. Design and Methods. The binding affinity of autoantibodies in patients' sera (T2DM, $n=100 ; \mathrm{ATH}, n=100$; and T2DM-ATH, $n=100)$ and isolated immunoglobulin G (IgG) against native fibrinogen (N-Fib) and MG-Fib to healthy subjects (HS, $n=50)$ was accessed by direct binding ELISA. The results of direct binding were further validated by competitive/inhibition ELISA. Moreover, AGE detection, ketoamines, protein carbonyls, hydroxymethylfurfural (HMF), thiobarbituric acid reactive substances (TBARS), and carboxymethyllysine (CML) concentrations in patients' sera were also determined. Furthermore, free lysine and free arginine residues were also estimated. Results. The high binding affinity was observed in 54\% of T2DM, 33\% of ATH, and 65\% of T2DM-ATH patients' samples with respect to healthy subjects against MG-Fib antigen in comparison to N-Fib $(p<0.05$ to $p<0.0001)$. HS sera showed nonsignificant binding $(p>0.05)$ with N-Fib and MG-Fib. Other biochemical parameters were also found to be significant $(p<0.05)$ in the patient groups with respect to the HS group. Conclusions. These findings in the future might pave a way to authenticate fibrinogen as a biomarker for the early detection of diabetes-associated micro- and macrovascular complications.
\end{abstract}

\section{Introduction}

In diabetes mellitus, hyperglycemia-mediated nonenzymatic glycosylation or glycation of biological macromolecules (proteins, lipids, and DNA) plays a critical role in the pathogenesis of secondary complications especially micro- and macrovascular complications $[1,2]$. During glycation, free carbonyl groups $(>\mathrm{C}=\mathrm{O})$ of reducing sugars bind with free amino $\left(-\mathrm{NH}_{2}\right)$ groups at the $\mathrm{N}$-terminal of proteins, $\varepsilon$-amino group of lysine, guanidino group of arginine, and imidazole group of histidine to form reversible aldimines or Schiff s bases $[3,4]$. Schiff's bases via keto-enol tautomerism and acid-base catalysis convert to irreversible ketoamines or Amadori products, and the latter undergo rearrangements, 
dehydrations, and cyclizations to form heterogeneous molecular adducts, known as advanced glycation end products (AGEs) $[5,6]$.

Glycoxidation of glycation-adducts and autoxidation of glucose lead to the generation of reactive oxygen species (ROS) such as superoxide radicals $\left(\mathrm{O}_{2}{ }^{*}\right)$ via transition metal-catalyzed reaction and hydroxyl radicals $\left({ }^{\circ} \mathrm{OH}\right)$ via Fenton's reaction, thereby inducing oxidative stress $[7,8]$. Besides ROS generation, both autoxidation and glycoxidation give rise to aldehyde and/or ketone groups possessing highly electronegative dicarbonyls, called reactive carbonyl species (RCS), i.e., glyoxal (GO), methylglyoxal (MG), and 3deoxyglucosone (3-DG) $[9,10]$. Moreover, ROS-mediated oxidative stress induces lipid peroxidation and oxidation of amino acids, which result in the generation of RCS, especially MG. Furthermore, channelization of excess glucose in glycolysis and the polyol pathway leads to the generation of MG $[11,12]$. In human plasma, the physiological concentration of $\mathrm{MG}$ is $50-150 \mathrm{nM}[13,14]$. When this concentration goes beyond the normal and remains unchecked, the cellular system suffers carbonyl stress [15]. Furthermore, MG similarly glycates proteins as that of reducing sugars, but this time more violently, and induces a glycation-oxidation vicious cycle, thus accelerating glycative, oxidative, and carbonyl stress, the necessary outcome of which is AGE formation [16-18].

Fibrinogen $(340 \mathrm{kDa}$; half-life $\sim 4$ days; concentration 2 $4 \mathrm{mg} \mathrm{mL}^{-1}$ ) is an independent marker for vascular complications especially in atherosclerosis $[19,20]$. Elevated fibrinogen levels and glycative insult suffered by fibrinogen are among the factors that are responsible for atherosclerosis-mediated CVD risk in diabetic patients $[21,22]$. The glycemic control influences the survival of fibrinogen in circulation that reduces during hyperglycemia and normalizes during euglycemia [23]. This correlation results due to the extent of fibrinogen glycation, as the glycated fibrinogen, clear more rapidly than nonglycated fibrinogen [24]. It has been reported that glycated fibrinogen usually resides extravascularly whereas the nonglycated one is distributed intravascularly. The extravascular accumulation (in the endothelial cells) of fibrinogen-AGEs (Fib-AGEs) results in their binding to receptors, called receptor for AGEs (RAGE), thereby establishing an AGE-RAGE axis. This axis triggers a signaling cascade, which in turn promotes atherosclerosis via the activation of ERK, JNK, PI3K, $\mathrm{p} 38, \mathrm{NF}-\kappa \mathrm{B}$, and cAMP pathways. Thus, the increased uptake of glycated fibrinogen into vascular walls might contribute to the progression of atherosclerosis in diabetic patients [25]. Glycation causes structural modifications and functional alterations in fibrinogen molecule that ultimately results in the formation of a lysis-resistant dense and less porous fibrin network [26].

The lysine residues of fibrinogen play a central role in the formation and degradation (fibrinolysis) of the fibrin network. During blood coagulation, fibrinopeptides A and $B$ cleave from the fibrinogen molecule by the action of thrombin to form fibrin monomers. These monomers polymerize to form protofibrils and get interwoven to give rise to a fibrin network or clot. The tensile strength of the clot and its resistance to lysis are imparted by peptide bonds between glutamine and lysine residues. These peptide bonds between adjacent fibrin molecules are formed by the action of activation factor XIII [25]. The lysis of the fibrin network (clot) is carried either by activation factor XIII-induced cross-linkage at the lysine residue or by direct binding of plasminogen, tissue plasminogen activator (tPA), and plasminogen activator inhibitor-1 (PAI-1) to lysine residues in fibrin molecules [27, 28]. Thus, the glycation at lysine residues affects factor XIII cross-linkage, fibrin clot structure, and regulation of fibrinolysis. Glycated fibrinogen possesses higher clotting activity than nonglycated fibrinogen [29]. It has been reported that resistance to fibrinolysis increases with an increase in fibrinogen glycation [30]. The plasminolysis system that comprises plasminogen, tPA, and PIA-1 requires to bind at free lysine residues for lysis. However, lysine residues are engaged due to glycation and are not available for the process of fibrinolysis [31, 32].

Moreover, AGE accumulation results in the monocytemacrophage-mediated production of cytokines (IL-1 and IL-6), which in turn activates hepatocytes for the overproduction of fibrinogen $[33,34]$. Besides the expression of adhesion molecules on endothelial cells, AGEs also stimulate monocyte migration and cytokine secretion from macrophages [35]. Furthermore, AGEs increase the levels of endothelin-1 and decrease the levels of nitric oxide, thereby resulting in overall vasoconstriction [36]. Additionally, AGEs facilitate clot formation by suppressing fibrinolysis via the activation of plasminogen activator inhibitor-1 (PAI-1) [37].

Glycation of fibrinogen with MG results in structural modifications and exposure of neoepitopes that elicit immune responses in experimental animals [38]. Previous studies by our research group have revealed the presence of autoantibodies against d-ribose-glycated LDL, d-riboseglycated hemoglobin (Hb), and MG-glycated LDL in type 2 diabetes mellitus (T2DM) and its associated secondary complications [39-44]. Therefore, we hypothesized that MGmediated glycation of fibrinogen leads to the generation of autoantibodies (anti-MG-Fib-IgG) in a hyperglycemic state. The present study was designed to probe the prevalence of autoantibodies against MG-glycated fibrinogen (MG-Fib) in patients suffering from T2DM, atherosclerosis (ATH), and diabetic atherosclerosis (T2DM-ATH).

\section{Materials and Methods}

2.1. Materials. Fibrinogen, methylglyoxal, oxalic acid, thiobarbituric acid (TBA), trichloroacetic acid (TCA), sodium chloride $(\mathrm{NaCl})$, sodium hydroxide $(\mathrm{NaOH})$, phosphotungstic acid, bovine serum albumin (BSA), ammonium persulphate (APS), bis-acrylamide, and tetramethylethylenediamine (TEMED) were purchased from Hi-Media. Sodium dodecyl sulfate (SDS) and 2,4,-trinitrobenzene-1-sulphonic acid (TNBS) were obtained from G Biosciences, whereas ethyl acetate and dinitrophenylhydrazine (DNPH) were purchased from Rankem. Guanidium hydrochloride was obtained from S. D. Fine-Chem Limited, and hydrochloric acid ( $\mathrm{HCl})$ was purchased from Fisher Scientific. A protein A agarose column was purchased from Sigma, and polystyrene plates were obtained from Nunc (Denmark). Analytical grade chemicals 
were used in this study. All the solutions were filtered under aseptic conditions using a sterilized Puradisc ${ }^{\mathrm{TM}} 0.2 \mathrm{mM}$ syringe filter (Whatman, GE Healthcare UK Limited, UK).

2.1.1. Ethical Statement. The clinical study has been approved by the "Institutional Ethics Committee (IEC) of Integral Institute of Medical Sciences and Research" (IIMS\&R), Lucknow, U.P., India, with approval number IEC/IIMS\&R/2019/51.

2.1.2. Population Study and Collection of Sera. The study was performed on 350 serum samples from patients and healthy individuals. The study subjects were divided into four groups, i.e., (1) healthy subjects (HS, $n=50$ ), (2) type 2 diabetes mellitus (T2DM, $n=100)$, (3) atherosclerosis (ATH, $n=100$ ), and (4) diabetic atherosclerosis (T2DM-ATH, $n$ $=100$ ). Prior consent as per the guidelines of the Helsinki declaration was taken ahead of blood withdrawal from healthy subjects and patients. The blood samples were centrifuged at $4^{\circ} \mathrm{C}$ for 20 minutes at $2000 \mathrm{rpm}$. Isolated serum samples were incubated for $30 \mathrm{~min}$ at $56^{\circ} \mathrm{C}$ to inactivate complement proteins. Finally, $0.01 \%$ sodium azide was added to the sera, and the latter were stored at $-20^{\circ} \mathrm{C}$. Serum samples from the HS group were used as a control.

\subsubsection{Inclusion/Exclusion Criteria}

(1) Inclusion Criteria.

(a) Patients with T2DM, ATH, and T2DM-ATH

(b) Age group between 40 and 75 years with disease duration 5-15 years for all patients

\section{(1) Exclusion Criteria.}

(a) Patients suffering from any chronic disease other than T2DM, ATH, and T2DM-ATH

(b) Patients suffering from any acute or chronic infection

2.1.4. Biochemical Investigations. The clinical data of biochemical parameters such as BMI, WHR, blood pressure (systolic/diastolic), fasting blood glucose, HbA1c, total cholesterol (TC), triglycerides (TG), low-density lipoprotein (LDL), and high-density lipoprotein (HDL) were collected from the clinical laboratory of IIMS\&R, Lucknow, India.

\subsection{Methods}

2.2.1. Preparation of Antigen: Methylglyoxal-Glycated Fibrinogen. Human fibrinogen $\left(150 \mu \mathrm{g} \mathrm{mL}^{-1}\right)$ was treated with $10 \mathrm{mM}$ of methylglyoxal (MG) in $100 \mathrm{mM}$ sodium phosphate buffer saline (PBS) $(7.4 \mathrm{pH})$ under sterile conditions. Sodium azide $(0.05 \%)$ was added to reaction solutions to inhibit fungal and microbial growth. Both the native fibrinogen (N-Fib) and MG-glycated fibrinogen (MG-Fib) were incubated at $37^{\circ} \mathrm{C}$ for 7 days. Extensive dialysis was performed to get rid of unbound constituents. The MGinduced structural modifications in MG-Fib against N-Fib were characterized by different biochemical and biophysical techniques as previously published by us [38].

2.2.2. Direct Binding ELISA of Patients' Sera. Direct binding ELISA was accomplished on a 96-well polystyrene plate to validate titer of autoantibodies that might have risen against $\mathrm{N}-\mathrm{Fib}$ and MG-Fib in T2DM, ATH, and T2DM-ATH patients [45]. The wells were coated with $10 \mu \mathrm{g}$ of $\mathrm{N}$-Fib and MG-Fib in $100 \mu \mathrm{L}$ of $0.5 \mathrm{~mol} \mathrm{~L}^{-1}$ carbonate/bicarbonate buffer having a $\mathrm{pH}$ of 9.6. The samples were coated in triplicate, and half part of the ELISA plate was used as the control. The plates were incubated at $37^{\circ} \mathrm{C}(2 \mathrm{~h})$ and were left at $4^{\circ} \mathrm{C}$ overnight. After overnight incubation, washing was done thrice with TBS-T $(20 \mathrm{mM}$ Tris, $2.68 \mathrm{mM} \mathrm{KCL}$, and $150 \mathrm{mM} \mathrm{NaCl}$ (pH 7.4) having 0.05\% Tween-20). The unoccupied sites of wells were blocked with $2.5 \%$ bovine serum albumin (BSA), and the plates were again incubated at $37^{\circ} \mathrm{C}(4 \mathrm{~h})$. After incubation, plates were again washed thrice with TBS-T. Serially diluted sera $(1: 100)$ from control and patients were appended to each well $(100 \mu \mathrm{L}$ per well), and the plates were incubated at $37^{\circ} \mathrm{C}(2 \mathrm{~h})$. After incubation, the wells were again washed thrice with TBS-T and were coated with anti-human IgG-alkaline phosphatase $(1: 2000)$ and left for two hours at $37^{\circ} \mathrm{C}$. After incubation, washing was done with TBS-T and the wells were coated with $p$ nitrophenylphosphate ( $\mathrm{pNPP}$ ) substrate. The reading was taken at $410 \mathrm{~nm}$ on an ELISA plate reader, and the results were calculated as a mean of $A_{\text {test }}-A_{\text {control }}$.

2.2.3. IgG Isolation from $H S$ and T2DM, ATH, and T2DMATH Patients' Sera. The protein A agarose affinity column was used to isolate immunoglobulin $\mathrm{G}$ ( $\mathrm{IgG}$ ) from HS and T2DM, ATH, and T2DM-ATH patients' sera that showed high autoantibody titer in the direct binding ELISA. Equal volumes $(0.5 \mathrm{~mL})$ of serum and phosphate buffer saline (0.1 M; pH 7.4) were mixed and passed through the affinity column 3-5 times. The bound IgG was eluted by $0.058 \%$ acetic acid in $0.85 \%$ sodium chloride solution. The $2 \mathrm{~mL}$ eluted solution containing IgG was collected in $1 \mathrm{~mL}$ of $1 \mathrm{M}$ Tris$\mathrm{HCl}$ ( $\mathrm{pH}$ 8.5) buffer. The concentration of isolated IgG was determined by using the molar extinction coefficient of 2.1 $\times 10^{5} \mathrm{M}^{-1} \mathrm{~cm}^{-1}$ at $280 \mathrm{~nm}$ wavelength [46].

2.2.4. Direct Binding ELISA of Isolated IgG. Direct binding ELISA was performed to elucidate the binding affinity of IgG that was purified from T2DM, ATH, and T2DM-ATH patients' sera. Polystyrene plates were coated with the $\mathrm{N}$ Fib and MG-Fib $(10 \mu \mathrm{g} / 100 \mu \mathrm{L})$. The experimental procedure was the same as described for ELISA of the patients' sera (Section 2.2.2) except that purified IgG was used in place of sera [47].

2.2.5. Competitive ELISA. Competitive/inhibition ELISA was performed to ensure antigen-binding specificity of autoantibodies that were generated against N-Fib and MG-Fib in patients' sera. Immune complexes were prepared by mixing antigens (N-Fib and MG-Fib) in $0.1,0.5,1,5,10,20,50$, and $100 \mu \mathrm{g} \mathrm{mL}^{-1}$ concentrations with a constant amount of purified autoantibodies and were incubated at $37^{\circ} \mathrm{C}$ for two hours and then left at $4^{\circ} \mathrm{C}$ overnight. Native immune 
complexes (N-Fib+N-Fib-IgG) and glycated immune complexes (MG-Fib+MG-Fib-IgG) were used as inhibitors. All steps were the same as discussed earlier in the direct binding ELISA except that immune complexes were used as primary antibodies to be coated in place of sera or IgG [48]. Percent inhibition was calculated as

$$
\text { Percent inhibition }(\%)=\frac{1 \text {-inhibited }}{\text { Uninhibited }} \times 100 \text {. }
$$

2.2.6. Nitroblue Tetrazolium (NBT) Reduction Assay. The nitroblue tetrazolium (NBT) reduction assay was performed to detect ketoamines in the sera of HS and T2DM, ATH, and T2DM-ATH patients. The serum samples $(20 \mu \mathrm{L})$ were mixed with $180 \mu \mathrm{L}$ of $\mathrm{Na}_{2} \mathrm{CO}_{3} / \mathrm{NaHCO}_{3}$ buffer $(100 \mathrm{mM}$, $\mathrm{pH} 10.8$ ) containing $0.25 \mathrm{mM}$ NBT. The mixtures were incubated at $37^{\circ} \mathrm{C}$ until the color changed from yellow to purple. The absorbance was recorded at $525 \mathrm{~nm}$, and the molar extinction coefficient of $12,640 \mathrm{M}^{-1} \mathrm{~cm}^{-1}$ was used to calculate ketoamine concentration $\left(\mathrm{nM} \mathrm{mL}^{-1}\right)[49,50]$.

2.2.7. Fluorescence Spectroscopy. Fluorescence spectroscopy was performed to detect fluorescent AGEs in the sera of HS and T2DM, ATH, and T2DM-ATH patients. The $0.1 \mathrm{~mL}$ of serum was mixed with $0.4 \mathrm{~mL}$ of $100 \mathrm{mM}$ PBS of $\mathrm{pH}$ 7.4. The sample was excited at $370 \mathrm{~nm}$, and the emission was recorded between 400 and $500 \mathrm{~nm}$ on an Agilent Cary Eclipse fluorescence spectrophotometer at $25 \pm 0.2^{\circ} \mathrm{C}$ in a $1 \mathrm{~cm}$ path length quartz cuvette. The slit width was set at $5 \mathrm{~nm}[51,52]$. The percent increase in fluorescence intensity (F.I.) was calculated by the following equation:

$\%$ Increase in F.I. $=\frac{\text { F.I.of patient' } s \text { serum }- \text { F.I.of HS serum }}{\text { F.I.of patient }{ }^{\prime} \text { s serum }} \times 100$.

2.2.8. Protein Carbonyl Estimation. Protein carbonyl estimation in the sera of HS and T2DM, ATH, and T2DM-ATH patients was performed by using 2,4-dinitrophenylhydrazine (DNPH). The $0.1 \mathrm{~mL}$ of serum was mixed with $0.5 \mathrm{~mL}$ of $10 \mathrm{mM} \mathrm{DNPH}$ in $2.5 \mathrm{~N} \mathrm{HCL}$ and was incubated at room temperature for 1 hour. After incubation, $0.5 \mathrm{~mL}$ of TCA $(20 \% v / v)$ was added and the mixture was centrifuged at $10,000 \mathrm{rpm}$ for 10 minutes to precipitate DNP hydrazones. The precipitated pellets were extensively washed three times with $0.5 \mathrm{~mL}$ of ethanol-ethyl acetate solution $(1: 1 \mathrm{v} / \mathrm{v})$ to relieve surplus DNPH. The pellets were then dissolved in $0.25 \mathrm{~mL}$ of $6 \mathrm{M}$ guanidium hydrochloride solution. Protein carbonyl concentration $\left(\mathrm{nM} \mathrm{mg}^{-1}\right)$ was evaluated by using a molar extinction coefficient of $22,000 \mathrm{M}^{-1} \mathrm{~cm}^{-1}$ at $370 \mathrm{~nm}$ wavelength $[53,54]$.

2.2.9. Hydroxymethylfurfural (HMF) Estimation. Hydroxymethylfurfural (HMF) estimation in the sera of HS and T2DM, ATH, and T2DM-ATH patients was performed by using thiobarbituric acid (TBA). The $0.1 \mathrm{~mL}$ of serum was mixed with $0.1 \mathrm{~mL}$ of $1 \mathrm{M}$ oxalic acid and was left for 1 hour in the water bath. After cooling at room temperature, trichloroacetic acid (40\%) was mixed in the solutions. The pre- cipitate was discarded, and the thiobarbituric acid (TBA) was added to the supernatant and was incubated at $37^{\circ} \mathrm{C}$ for half an hour. After the appearance of color, HMF concentration $\left(\mathrm{nM} \mathrm{mL}^{-1}\right)$ was evaluated by using the molar extinction coefficient of $4 \times 104 \mathrm{M}^{-1} \mathrm{~cm}^{-1}$ at $443 \mathrm{~nm}$ wavelength [55].

2.2.10. Thiobarbituric Acid Reactive Substance (TBARS) Estimation. TBARS estimation in the sera of HS and T2DM, ATH, and T2DM-ATH patients was performed by adding $\mathrm{H}_{2} \mathrm{SO}_{4}$ and phosphotungstic acid. The $0.1 \mathrm{~mL}$ of serum was mixed with $0.4 \mathrm{~mL}$ of $0.083 \mathrm{~N} \mathrm{H}_{2} \mathrm{SO}_{4}$ and $0.2 \mathrm{~mL}$ of $10 \%$ phosphotungstic acid. The solutions were left at room temperature for 5 minutes and then centrifuged at $3000 \mathrm{rpm}$ for 10 minutes. The pellets were mixed with $2 \mathrm{~mL}$ of distilled water, and $0.5 \mathrm{~mL}$ of TBA reagent (a mixture containing $0.37 \%$ aqueous TBA, $15 \%$ aqueous TCA, and $0.25 \mathrm{~N} \mathrm{HCl}$ ) was added. The solutions were heated for 1 hour at $95^{\circ} \mathrm{C}$, and centrifugation at $3000 \mathrm{rpm}$ for 10 minutes was performed to collect the supernatant. The supernatant was left to cool at room temperature. TBARS concentration $\left(\mu \mathrm{M} \mathrm{mL}^{-1}\right)$ was evaluated by using the molar extinction coefficient of $1.56 \times 10^{5} \mathrm{M}^{-1} \mathrm{~cm}^{-1}$ at $532 \mathrm{~nm}$ wavelength [56].

2.2.11. Free Lysine Residue Estimation. Free lysine residue estimation in the sera of HS and T2DM, ATH, and T2DM-ATH patients was performed by using 2,4,6-trinitrobenzene sulphonic acid (TNBS). The $0.1 \mathrm{~mL}$ serum was mixed with $0.1 \mathrm{~mL}$ of $\mathrm{NaHCO}_{3}$ buffer ( $\left.4 \% w / v, \mathrm{pH} 8.5\right)$. The $0.1 \mathrm{~mL}$ of $0.1 \%$ TNBS was added to the solutions, and the latter were incubated at $40^{\circ} \mathrm{C}$ for 2 hours. After incubation, $0.45 \mathrm{~mL}$ of HCL was added and again the solutions were incubated at $110^{\circ} \mathrm{C}$ for 90 minutes. After cooling the solutions, centrifugation was done at $3000 \mathrm{rpm}$ for 10 minutes. The supernatant was mixed with $1 \mathrm{~mL}$ ether to remove the $\alpha$-TNP amino complex, and finally the solutions were kept on a water bath till the evaporation of excess ether. The absorbance was taken at $420 \mathrm{~nm}[57,58]$.

2.2.12. Free Arginine Residue Estimation. Free arginine residue estimation in the sera of HS and T2DM, ATH, and T2DM-ATH patients was performed by using 9,10-phenanthrenequinone. The $0.1 \mathrm{~mL}$ serum was mixed with $0.3 \mathrm{~mL}$ of $200 \mu \mathrm{M}$ of phenanthrenequinone (dissolved in ethanol). After mixing, $0.5 \mathrm{~mL}$ of $2 \mathrm{~N} \mathrm{NaOH}$ was added and the solutions were left for $60 \mathrm{~min}$ at $30^{\circ} \mathrm{C}$. After cooling at room temperature, $0.5 \mathrm{~mL}$ of $1.2 \mathrm{M} \mathrm{HCl}$ was added and the fluorescence was taken on the Agilent Cary Eclipse fluorescence spectrophotometer at $25 \pm 0.2^{\circ} \mathrm{C}$ in a $1 \mathrm{~cm}$ path length quartz cuvette. The samples were excited at $312 \mathrm{~nm}$, and the emission was recorded at $395 \mathrm{~nm}$ [59].

2.2.13. Detection of Nonfluorogenic AGE: Carboxymethyllysine $(C M L)$. Carboxymethyllysine (CML) is a nonfluorogenic AGE that was detected in the sera of HS and T2DM, ATH, and T2DM-ATH patients by using a sandwich ELISA kit from Bioassay Technology Laboratory (Korain Biotech Co. Ltd.). The serum was diluted to $1: 100$ ratios with PBS $(100 \mathrm{mM}$; $\mathrm{pH}$ 7.4). The diluted samples were added to the wells that were already coated with monoclonal antibodies against CML. 
After incubating for 15 minutes, the anti-CML antibody was added. The excess anti-CML antibody was removed during the washing step. Later on, streptavidin-HRP was added to the wells that bound to the anti-CML antibodies. After incubating for 15 minutes, washing was done to remove unbound streptavidin-HRP. Finally, the substrate was added that imparted color in the proportion of CML present in the serum samples. After the appearance of color, the reaction was terminated by adding an acidic stop solution and the absorbance was read at $450 \mathrm{~nm}[60]$.

2.2.14. Statistical Analysis. The data for the present study was presented as mean $\pm \mathrm{SD}$, and the statistical significance was determined by one-way ANOVA followed by the post hoc test, Tukey's multiple comparison test, and Dunnett $t$ -test using the Statistical Package for the Social Sciences (SPSS) version 20.0. The coefficient of variation within groups (HS, T2DM, ATH, and T2DM-ATH) was analyzed by an unpaired $t$-test. The value of $p<0.05$ was considered statistically significant. The scattered plots were plotted using GraphPad Prism version 5.0.1.

\section{Results}

3.1. Biophysical and Biochemical Characterization of Methylglyoxal-Glycated Fibrinogen. The in vitro methylglyoxal- (MG-) mediated glycation of fibrinogen protein results in secondary and tertiary level structural modifications as illustrated in our previous study [38]. The results of physicochemical characterization of native fibrinogen (N-Fib) and MG-glycated fibrinogen (MG-Fib) are summarized in Table 1.

3.2. Evaluation of Clinical Parameters in Patients and Healthy Subjects. The clinical parameters of $\mathrm{HS}$ and T2DM, ATH, and T2DM-ATH patients vary significantly in age and gender distribution at the time of testing. Gender difference does not play a significant role in the levels of MG-glycated fibrinogen levels in the patients. However, factors besides glycation such as smoking, alcohol consumption, disturbed daily routine, lifestyle, obesity, depression, hypertension, and stress are responsible for the genderdependent development of cardiovascular diseases. Blood pressure (mmHg), both systolic (SST) and diastolic (DST), was moderately higher in T2DM patients $(125 \pm 12 / 78 \pm 9)$ but was in the normal range, whereas in ATH $(164 \pm 22$ / $106 \pm 10)$ and T2DM-ATH $(180 \pm 9.5 / 110 \pm 12)$ patients, the blood pressures were significantly higher with respect to HS $(120 \pm 15 / 65 \pm 7)$. The body mass index (BMI) of HS $\left(20.5 \pm 1.5 \mathrm{~kg} \mathrm{~m}^{-2}\right)$ and ATH patients $\left(21.6 \pm 2.0 \mathrm{~kg} \mathrm{~m}^{-2}\right)$ was almost similar. However, in T2DM $(26.55 \pm 2.13 \mathrm{~kg}$ $\left.\mathrm{m}^{-2}\right)$ and T2DM-ATH $\left(27.3 \pm 2.3 \mathrm{~kg} \mathrm{~m}^{-2}\right)$ patients, BMI was significantly higher with respect to $\mathrm{HS}$. The HbA1c was found to be significantly higher in T2DM $(6.6 \pm 1.92 \%)$ and T2DM-ATH $(7.0 \pm 2.81 \%)$ patients than in ATH $(4.4 \pm 0.22 \%)$ patients and HS $(4.5 \pm 0.14 \%)$. The fasting glucose levels ( $\mathrm{mg} \mathrm{dL}^{-1}$ ) were significantly higher in T2DM $(196 \pm 8.2)$ and T2DM-ATH $(222 \pm 16)$ patients with respect to ATH $(90 \pm 6)$ and HS $(88 \pm 8)$. The lipid profiles of ATH and T2DM-ATH patients vary significantly with respect to T2DM and HS groups. The data of clinical parameters are presented in Table 2.

3.3. Direct Binding ELISA with Sera of T2DM, ATH, and T2DM-ATH Patients. Direct binding ELISA was performed for the screening of HS and T2DM, ATH, and T2DMATH patients' sera against N-Fib and MG-Fib. The sera of the HS group were used as the control. The mean absorbance values at $410 \mathrm{~nm}$ of all the four groups were as follows: HS $(0.204 \pm 0.021 \quad(0.175-0.234)), \quad$ T2DM $\quad(0.215 \pm 0.024$ (0.192-0.288)), ATH (0.212 $\pm 0.029(0.180-0.282))$, and T2DM-ATH $(0.220 \pm 0.021(0.200-0.295))$. The autoantibodies in the sera of T2DM and T2DM-ATH groups showed significant binding against $\mathrm{N}-\mathrm{Fib}$ with respect to the $\mathrm{HS}$ group, i.e., $p<0.05$ and $p<0.001$, respectively. However, between the HS group and the ATH group, the binding of autoantibodies against $\mathrm{N}$-Fib was not significant $(p>0.05)$. Moreover, ATH and T2DM-ATH groups showed significance $(p<0.05)$ among them. The coefficient of variation in HS and T2DM, ATH, and T2DM-ATH groups was 10.63\%, 11.50\%, $13.78 \%$, and $9.72 \%$, respectively (Figure $1(\mathrm{a})$ ).

The autoantibodies in the sera of T2DM (54\%), ATH (33\%), and T2DM-ATH (65\%) exhibited higher binding affinity for MG-Fib. The mean absorbance values at $410 \mathrm{~nm}$ of all the four groups were as follows: HS $(0.211 \pm$ $0.018(0.182-0.240))$, T2DM $(0.513 \pm 0.209$ (0.264-0.908)), ATH $(0.424 \pm 0.170 \quad(0.280-0.846))$, and T2DM-ATH $(0.581 \pm 0.223(0.266-0.970))$.

In the T2DM group, out of 54 samples that showed significant binding against MG-Fib, 17 samples were highly significant $(p<0.0001), 13$ samples were very significant $(p<0.001)$, and 24 samples were significant $(p<0.05)$. In the ATH group, out of 33 samples, 7 samples were highly significant $(p<0.0001), 12$ samples were very significant $(p<0.001)$, and 14 samples were significant $(p<0.05)$. Similarly, in the T2DM-ATH group, out of 65 samples, 21 samples were highly significant $(p<0.0001), 20$ samples were very significant $(p<0.001)$, and 24 samples were significant $(p<0.05)$. With respect to HS, all the three patient groups exhibited high statistical significance $(p<0.0001)$. However, between T2DM and T2DM-ATH, the significance level was $p>0.05$. The coefficient of variation in HS, T2DM, ATH, and T2DM-ATH groups was $8.81 \%, 40.70 \%, 40.09 \%$, and $38.37 \%$, respectively (Figure $1(\mathrm{~b})$ ). The statistical data are summarized in Table 3.

Note that after the screening, all the biophysical and biochemical experimentations were performed on 54 T2DM, 33 ATH, and 65 T2DM-ATH serum samples.

3.4. Direct Binding ELISA with IgG of T2DM, ATH, and T2DM-ATH Patients. Direct binding ELISA with purified IgG from patients' sera was performed to further validate the binding affinity of autoantibodies against $\mathrm{N}$-Fib and MG-Fib. The IgG was purified from the serum samples that showed higher binding during screening (Section 3.3). Direct binding of purified IgG with N-Fib showed statistical significance in T2DM $(p<0.05)$, ATH $(p<0.05)$, and T2DM-ATH $(p<0.001)$ with respect to the HS group 
TABLE 1: Comparative physicochemical characterization of fibrinogen protein upon glycation with $5 \mathrm{mM}$ and $10 \mathrm{mM}$ of methylglyoxal (MG).

\begin{tabular}{|c|c|c|c|}
\hline \multirow{2}{*}{ Characterization } & \multicolumn{3}{|c|}{ Fibrinogen } \\
\hline & Native & $5 \mathrm{mM} \mathrm{MG}$ & $10 \mathrm{mM} \mathrm{MG}$ \\
\hline $\mathrm{UV}-\mathrm{V}$ is $(280 \mathrm{~nm})$ & $0.231 \pm 0.05$ & $\begin{array}{c}1.134 \pm 0.24 \\
79 \% \uparrow\end{array}$ & $\begin{array}{c}1.268 \pm 0.42 \\
81 \% \uparrow\end{array}$ \\
\hline Ketoamines $\left(\mathrm{nM} \mathrm{mL}^{-1}\right)$ & $4.43 \pm 0.76$ & $\begin{array}{c}38.68 \pm 4.3 \\
88 \% \uparrow\end{array}$ & $\begin{array}{c}46.28 \pm 6.9 \\
90 \% \uparrow\end{array}$ \\
\hline AGEs $\left(\lambda_{\mathrm{ex}}-370 ; \lambda_{\mathrm{em}}-460\right)$ & $3.17 \pm 0.5$ & $\begin{array}{c}12.67 \pm 3.3 \\
74 \% \uparrow\end{array}$ & $\begin{array}{c}15.73 \pm 4.7 \\
79 \% \uparrow\end{array}$ \\
\hline Intrinsic fluorescence $\left(\lambda_{\mathrm{ex}}-280 ; \lambda_{\mathrm{em}}-340\right)$ & $304 \pm 41$ & $\begin{array}{l}77.31 \pm 10 \\
74 \% \downarrow\end{array}$ & $\begin{array}{l}55.30 \pm 6.2 \\
81 \% \downarrow\end{array}$ \\
\hline Circular dichroism (mdeg) & $-10.23 \pm 0.1$ & $-15.4 \pm 1.3$ & $-17.3 \pm 1.7$ \\
\hline FTIR $\left(\mathrm{cm}^{-1}\right)$ & $\begin{array}{l}1636 \\
3438\end{array}$ & $\begin{array}{l}1640 \\
3442\end{array}$ & $\begin{array}{l}1638 \\
3444\end{array}$ \\
\hline Carbonyl content $\left(\mathrm{nM} \mathrm{mg}^{-1}\right)$ & $40 \pm 10$ & $\begin{array}{c}147 \pm 18 \\
72 \% \uparrow\end{array}$ & $\begin{array}{c}219 \pm 31 \\
81 \% \uparrow\end{array}$ \\
\hline $\operatorname{HMF}\left(\mathrm{nmol} \mathrm{mL} L^{-1}\right)$ & $1.2 \pm 0.2$ & $\begin{array}{c}5.8 \pm 0.78 \\
79 \% \uparrow\end{array}$ & $\begin{array}{c}7.2 \pm 1.6 \\
83 \% \uparrow\end{array}$ \\
\hline TBARS $\left(\mu \mathrm{M} \mathrm{mL}^{-1}\right)$ & $2.2 \pm 0.3$ & $\begin{array}{c}9 \pm 0.98 \\
75 \% \uparrow\end{array}$ & $\begin{array}{c}11.6 \pm 1.2 \\
81 \% \uparrow\end{array}$ \\
\hline Free lysine $(420 \mathrm{~nm})$ & $0.423 \pm 0.02$ & $\begin{array}{c}0.098 \pm 0.033 \\
76 \% \downarrow\end{array}$ & $\begin{array}{c}0.079 \pm 0.02 \\
81 \% \downarrow\end{array}$ \\
\hline Free arginine $\left(\lambda_{\mathrm{ex}}-312 ; \lambda_{\mathrm{em}}-395\right)$ & $149 \pm 18$ & $\begin{array}{c}69.69 \pm 13.4 \\
53 \% \downarrow\end{array}$ & $\begin{array}{c}57.73 \pm 9.1 \\
61 \% \downarrow\end{array}$ \\
\hline $\mathrm{CML}\left(\mathrm{nM} \mathrm{mg}^{-1}\right)$ & $183 \pm 28$ & $\begin{array}{c}631 \pm 76 \\
70 \% \uparrow\end{array}$ & $\begin{array}{c}657 \pm 54 \\
72 \% \uparrow\end{array}$ \\
\hline $\operatorname{ThT}\left(\lambda_{\mathrm{ex}}-440 ; \lambda_{\mathrm{em}}-485\right)$ & $32.31 \pm 9.1$ & $\begin{array}{c}107 \pm 11 \\
69 \% \uparrow\end{array}$ & $\begin{array}{c}131 \pm 20 \\
75 \% \uparrow\end{array}$ \\
\hline
\end{tabular}

TABLE 2: Clinical and metabolic parameters of the T2DM, ATH, and T2DM-ATH patients.

\begin{tabular}{|c|c|c|c|c|}
\hline Characteristics & HS & T2DM & ATH & T2DM-ATH \\
\hline Sample size & 50 & 100 & 100 & 100 \\
\hline Age (years) & $30 \pm 8$ & $53.4 \pm 11.4$ & $59 \pm 7.1$ & $55.4 \pm 10.24$ \\
\hline Gender (M/F) & $25 / 25$ & $67 / 33$ & $82 / 18$ & $78 / 12$ \\
\hline $\mathrm{DD}$ (years) & - & $7.6 \pm 2.3$ & $8.1 \pm 4.2$ & $9.2 \pm 5.2$ \\
\hline BMI $\left(\mathrm{kg} \mathrm{m}^{-2}\right)$ & $20.5 \pm 1.5$ & $26.55 \pm 2.13^{* *}$ & $21.6 \pm 2^{*}$ & $27.3 \pm 2.3^{* *}$ \\
\hline $\mathrm{BP}(\mathrm{SST})(\mathrm{mmHg})$ & $120 \pm 15$ & $125 \pm 12^{\dagger}$ & $164 \pm 22.0^{* *}$ & $180 \pm 9.5^{* *}$ \\
\hline $\mathrm{BP}$ (DST) (mmHg) & $65 \pm 7$ & $78 \pm 9^{\dagger}$ & $106 \pm 10^{* *}$ & $110 \pm 12^{* *}$ \\
\hline Glucose: fasting $\left(\mathrm{mg} \mathrm{dL}^{-1}\right)$ & $88 \pm 8$ & $196 \pm 8.2^{* *}$ & $90 \pm 6$ & $222 \pm 16^{* * *}$ \\
\hline HbAlc (\%) & $4.5 \pm 0.14$ & $6.6 \pm 1.9^{* *}$ & $4.4 \pm 0.2$ & $7.1 \pm 2.9^{* * *}$ \\
\hline $\mathrm{TC}\left(\mathrm{mg} \mathrm{dL}^{-1}\right)$ & $160 \pm 2.1$ & $175 \pm 2.3^{\dagger}$ & $308 \pm 3.2^{* * *}$ & $284 \pm 10.4^{* *}$ \\
\hline $\mathrm{LDL}\left(\mathrm{mg} \mathrm{dL}^{-1}\right)$ & $144 \pm 4.11$ & $147 \pm 0.1^{\dagger}$ & $244 \pm 2.5^{* * *}$ & $246 \pm 4.4^{* *}$ \\
\hline $\mathrm{HDL}\left(\mathrm{mg} \mathrm{dL}^{-1}\right)$ & $48 \pm 1.8$ & $37.8 \pm 3.9^{\dagger}$ & $33 \pm 1.3^{* *}$ & $27 \pm 4.6^{* *}$ \\
\hline $\mathrm{TG}\left(\mathrm{mg} \mathrm{dL}^{-1}\right)$ & $176 \pm 4.1$ & $184 \pm 6.8^{*}$ & $300 \pm 3.3^{* * *}$ & $204 \pm 5.8^{* *}$ \\
\hline
\end{tabular}

Values are expressed as mean \pm SD: significance/nonsignificance level: ${ }^{\dagger} p>0.05$ (not significant), ${ }^{*} p<0.05$ (significant), ${ }^{* *} p<0.001$ (highly significant), and ${ }^{* * *} p<0.0001$ (extremely significant). All the parameters were tested by one-way ANOVA followed by the post hoc test and Dunnett $t$-test using the Statistical Package for the Social Sciences (SPSS) package, version 20.0. HS: healthy subjects; M/F: male/female; BMI: body mass index; DD: duration of disease; SST: systolic; DST: diastolic; HbA1c: glycosylated hemoglobin; TC: total cholesterol; LDL: low-density lipoprotein; HDL: high-density lipoprotein; TG: triglycerides. 


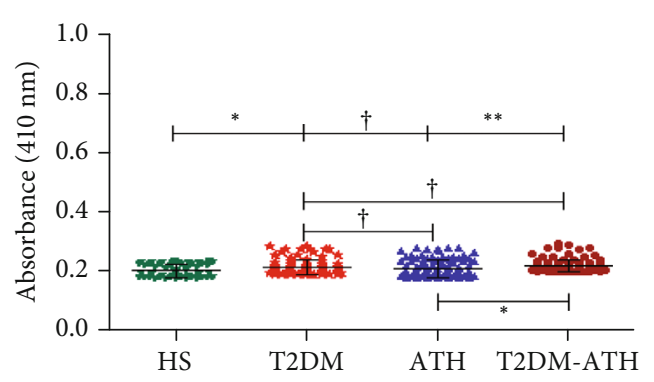

(a)

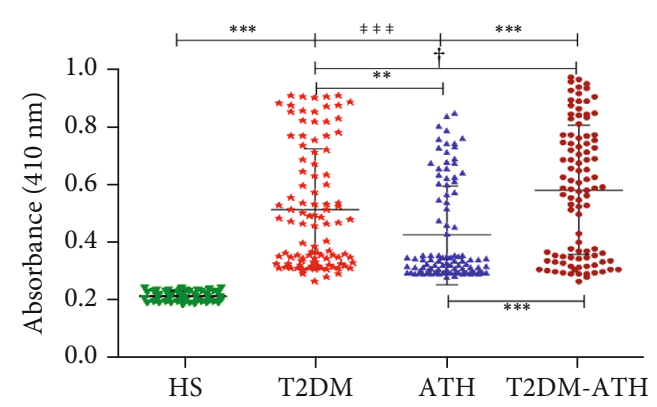

(b)

Figure 1: Direct binding ELISA of serum autoantibodies. (a) Direct binding ELISA of serum autoantibodies from diabetic (T2DM, $n=100$ ), atherosclerosis (ATH, $n=100$ ), and diabetic atherosclerosis (T2DM-ATH, $n=100$ ) patients with respect to healthy subjects (HS, $n=50$ ) against N-Fib $\left(10 \mu \mathrm{g} \mathrm{mL}^{-1}\right)$. T2DM $\left({ }^{*} p<0.05\right)$ and T2DM-ATH $\left({ }^{* *} p<0.001\right)$ groups show significant binding with respect to HS. The ATH group shows a nonsignificant $\left({ }^{\dagger} p>0.05\right)$ binding with respect to HS. Data are presented as mean \pm SD of three independent experiments. (b) Direct binding ELISA of serum autoantibodies from diabetic (T2DM, $n=100)$, atherosclerosis $(\mathrm{ATH}, n=100)$, and diabetic atherosclerosis (T2DM-ATH, $n=100)$ patients with respect to healthy subjects $(\mathrm{HS}, n=50)$ against $\mathrm{MG}-\mathrm{Fib}\left(10 \mu \mathrm{g} \mathrm{mL}{ }^{-1}\right)$. All the three patient groups showed significant binding with respect to HS and among themselves $\left({ }^{*} p<0.05,{ }^{* *} p<0.001\right.$, and $\left.{ }^{* * *} p<0.0001\right)$. Data are presented as mean \pm SD of three independent experiments.

(Figure 2(a)). However, between ATH and T2DM-ATH, the significance level was $p<0.05$. The mean absorbance values at $410 \mathrm{~nm}$ of all the four groups against N-Fib were as follows: HS (0.186 $\pm 0.022 \quad(0.163-0.235)), \quad$ T2DM $\quad(0.231 \pm 0.046$ (0.178-0.338)), ATH $(0.225 \pm 0.050 \quad(0.167-0.330))$, and T2DM-ATH $(0.238 \pm 0.050(0.180-0.370))$. The coefficient of variation in HS, T2DM, ATH, and T2DM-ATH groups was $12.16 \%, 20.19 \%, 22.31 \%$, and $21.85 \%$, respectively.

In the direct binding of IgG with MG-Fib, all the three patient groups were statistically significant $(p<0.0001)$ (Figure 2(b)). However, between the patient groups, the significance level varied. Between T2DM and T2DM-ATH, the value was $p>0.05$, whereas between T2DM and ATH groups, $p$ values were $<0.001$. Between ATH and T2DM-ATH groups, the statistical significance was higher $(p<0.0001)$. The mean absorbance values at $410 \mathrm{~nm}$ of all the four groups against MG-Fib were as follows: HS (0.187 \pm 0.019 (0.161-0.242)), T2DM $(0.834 \pm 0.088(0.661-1.040))$, ATH $(0.759 \pm 0.860$ (0.617-0.928)), and T2DM-ATH $(0.870 \pm 0.128 \quad(0.659-$ 1.20)). The coefficient of variation in HS, T2DM, ATH, and T2DM-ATH groups was $10.58 \%, 10.65 \%, 11.42 \%$, and $14.73 \%$, respectively (Figure 2(b)). The statistical data are summarized in Table 3.

3.5. Affinity of Isolated IgG to N-Fib and MG-Fib. The IgG concentration increases in T2DM and T2DM-ATH samples with respect to IgG isolated from HS. However, the concentration of IgG from ATH was higher than that from HS but was much lower in comparison to those from T2DM and T2DM-ATH subjects. To evaluate the concentration of IgG needed for the saturation of N-Fib and MG-Fib, direct binding ELISA was performed by isolated IgG from T2DM, ATH, and T2DM-ATH patients (Figure 3). The results revealed that the average saturation concentration of MGFib is $50 \mu \mathrm{g} \mathrm{mL}^{-1}$ of IgG from T2DM and T2DM-ATH patients. However, for the ATH group, the saturation concentration was $60 \mu \mathrm{g} \mathrm{mL}^{-1}$.
3.6. Competitive ELISA. Competitive/inhibition ELISA was performed to ensure antigen-binding specificity of purified a5utoantibodies that were generated against MG-Fib in patients' sera. The inhibition ELISA was done for $54 \mathrm{IgG}$ samples from the T2DM group, 33 IgG samples from the ATH group, and 65 IgG samples from the T2DM-ATH group, which were screened for greater affinity towards MGFib in the direct binding ELISA with sera (Section 3.3). The IgG samples from the HS group were used as a control.

The inhibition ELISA with the native immune complex $(\mathrm{N}-\mathrm{Fib}+\mathrm{IgG})$ showed statistical significance in the two groups of patients, i.e., T2DM $(p<0.001)$ and T2DM-ATH $(p<0.001)$ with respect to the HS group, whereas HS and ATH groups showed no significance $(p>0.05)$ (Figure 4(a)). At $20 \mu \mathrm{g} \mathrm{mL}^{-1}$ native inhibitor (N-Fib+N-Fib-IgG) concentration, the maximum \% inhibition for the T2DM group was found to be $24.50 \pm 5.77 \%$ (17.76-36.40); for the ATH group, it was $20.88 \pm 4.58(14.52-30.12)$; and for T2DM-ATH, it was $25.34 \pm 5.36$ (18.26-37.10). The coefficient of variation in HS, T2DM, ATH, and T2DM-ATH groups was 19.60\%, 23.56\%, $21.96 \%$, and $21.18 \%$, respectively.

However, with the glycated immune complex (MG-Fib + MG-Fib-IgG), all the three patient groups exhibited high statistical significance $(p<0.0001)$ with respect to the HS group (Figure $4(\mathrm{~b})$ ). The maximum $\%$ inhibition at $20 \mu \mathrm{g} \mathrm{mL}^{-1}$ inhibitor (MG-Fib+IgG) concentration for the T2DM group was $51.89 \pm 4.74$ (44.80-62.90); for the ATH group, it was $41.23 \pm 4.50(32.10-49.30)$; and for the T2DM-ATH group, it was found to be $59.25 \pm 6.43$ (44.7073.50). The coefficient of variation in HS, T2DM, ATH, and T2DM-ATH groups was $21.81 \%, 9.15 \%, 10.93 \%$, and $10.87 \%$, respectively. The statistical data are summarized in Table 3.

3.7. Ketoamine Estimation in Sera of HS and T2DM, ATH, and T2DM-ATH Patients. In the HS group, the mean ketoamine concentration was found to be $7.15 \pm 2.65 \mathrm{nM} \mathrm{mL}^{-1}$ 
TABLE 3: Statistical data showing direct binding and inhibition ELISA of sera and isolated IgG from HS, T2DM, ATH, and T2DM-ATH patients' groups against native fibrinogen (N-Fib) and methylglyoxal-glycated fibrinogen (MG-Fib).

\begin{tabular}{|c|c|c|c|c|c|c|c|}
\hline Disease & $N$ & Mean & $\mathrm{SD}$ & Min. & Max. & $\mathrm{CV}$ & $p$ value \\
\hline \multicolumn{8}{|c|}{ Direct binding ELISA (N-Fib/sera) } \\
\hline HS & 50 & 0.204 & 0.021 & 0.175 & 0.234 & $10.63 \%$ & $\ldots$. \\
\hline $\mathrm{T} 2 \mathrm{DM}$ & 100 & 0.215 & 0.024 & 0.192 & 0.288 & $11.50 \%$ & $p<0.05$ \\
\hline ATH & 100 & 0.212 & 0.029 & 0.180 & 0.282 & $13.78 \%$ & $p>0.05$ \\
\hline T2DM-ATH & 100 & 0.220 & 0.021 & 0.200 & 0.295 & $09.71 \%$ & $p<0.001$ \\
\hline \multicolumn{8}{|c|}{ Direct binding ELISA (MG-fib/sera) } \\
\hline HS & 50 & 0.211 & 0.018 & 0.182 & 0.240 & $8.81 \%$ & $\ldots$. \\
\hline $\mathrm{T} 2 \mathrm{DM}$ & 100 & 0.513 & 0.209 & 0.264 & 0.908 & $40.70 \%$ & $p<0.0001$ \\
\hline ATH & 100 & 0.424 & 0.170 & 0.280 & 0.846 & $40.09 \%$ & $p<0.0001$ \\
\hline T2DM-ATH & 100 & 0.581 & 0.223 & 0.266 & 0.970 & $38.37 \%$ & $p<0.0001$ \\
\hline \multicolumn{8}{|c|}{ Direct binding ELISA (N-Fib/IgG) } \\
\hline HS & 50 & 0.186 & 0.022 & 0.163 & 0.235 & $12.16 \%$ & - \\
\hline $\mathrm{T} 2 \mathrm{DM}$ & 54 & 0.231 & 0.046 & 0.178 & 0.338 & $20.19 \%$ & $p<0.05$ \\
\hline ATH & 33 & 0.225 & 0.050 & 0.167 & 0.330 & $22.31 \%$ & $p<0.05$ \\
\hline T2DM-ATH & 65 & 0.238 & 0.050 & 0.180 & 0.370 & $21.85 \%$ & $p<0.001$ \\
\hline \multicolumn{8}{|c|}{ Direct binding ELISA (MG-Fib/IgG) } \\
\hline HS & 50 & 0.187 & 0.019 & 0.161 & 0.242 & $10.58 \%$ & - \\
\hline $\mathrm{T} 2 \mathrm{DM}$ & 54 & 0.834 & 0.088 & 0.661 & 1.040 & $10.65 \%$ & $p<0.0001$ \\
\hline ATH & 33 & 0.759 & 0.086 & 0.617 & 0.928 & $11.42 \%$ & $p<0.0001$ \\
\hline T2DM-ATH & 65 & 0.870 & 0.128 & 0.659 & 1.200 & $14.73 \%$ & $p<0.0001$ \\
\hline \multicolumn{8}{|c|}{ Inhibition ELISA (N-Fib) } \\
\hline HS & 50 & 20.28 & 3.97 & 11.88 & 25.80 & $19.60 \%$ & - \\
\hline $\mathrm{T} 2 \mathrm{DM}$ & 54 & 24.50 & 5.77 & 17.76 & 36.40 & $23.56 \%$ & $p<0.001$ \\
\hline ATH & 33 & 20.88 & 4.58 & 14.52 & 30.12 & $21.96 \%$ & $p>0.05$ \\
\hline T2DM-ATH & 65 & 25.34 & 5.36 & 18.26 & 37.10 & $21.18 \%$ & $p<0.001$ \\
\hline \multicolumn{8}{|c|}{ Inhibition ELISA (MG-Fib) } \\
\hline HS & 50 & 19.78 & 4.18 & 12.40 & 26.70 & $21.18 \%$ & - \\
\hline $\mathrm{T} 2 \mathrm{DM}$ & 54 & 51.89 & 4.74 & 44.80 & 62.90 & $9.15 \%$ & $p<0.0001$ \\
\hline ATH & 33 & 41.23 & 4.50 & 32.10 & 49.30 & $10.93 \%$ & $p<0.0001$ \\
\hline T2DM-ATH & 65 & 59.25 & 6.43 & 44.70 & 73.50 & $10.87 \%$ & $p<0.0001$ \\
\hline
\end{tabular}

The data were presented as mean \pm SD, and the statistical significance level $(p<0.05)$ between HS, T2DM, ATH, and T2DM-ATH groups was determined by one-way ANOVA. All the three patient groups exhibit statistical significance $(p<0.0001)$ with the HS group. " $N$ " is the no. of subjects in each group, "Min." is the minimum value, "Max." is the maximum value, "SD" is the standard deviation, "CV" is the coefficient of variation, and " $p$ " is the level of significance. The upward arrows $(\uparrow)$ represent percent increase whereas downward arrows $(\downarrow)$ represent percent decrease in parameters with respect to the HS group.

(3.12-12.43), whereas in T2DM, ATH, and T2DM-ATH, the mean concentrations of ketoamines were higher up to $36.03 \pm 7.30$ (21.98-45.32), $27.52 \pm 7.16$ (16.04-35.19), and $39.83 \pm 7.86 \mathrm{nM} \mathrm{mL}^{-1}$ (25.51-52.62), respectively. The percent increase in ketoamines in T2DM, ATH, and T2DMATH groups with respect to healthy subjects was $80 \%$, $72 \%$, and $82 \%$, respectively (Figure 5 ). All the three patient groups exhibited high statistical significance $(p<0.0001)$ with respect to HS. Moreover, between the patient groups, the level of significance was from $p<0.001$ to $p<0.0001$. The coefficient of variation in HS, T2DM, ATH, and T2DM-ATH groups was $37.06 \%, 20.28 \%, 26.04 \%$, and
$19.73 \%$, respectively. The statistical data are summarized in Table 4.

3.8. AGE Detection in Sera of HS and T2DM, ATH, and T2DM-ATH Patients. Fluorescence spectroscopy was performed to detect fluorescent AGEs in HS and patient groups. In the HS group, the mean fluorescence intensity (F.I.) was found to be $16.03 \pm 4.78$ (a.u.) (9.34-25.47), whereas in T2DM, ATH, and T2DM-ATH, the mean F.I. were higher up to $65.37 \pm 12.31 \quad(47.28-83.54), 45.47 \pm 7.36$ (31.2360.49), and $71.90 \pm 13.13$ (a.u.) (42.21-88.61), respectively. The percent increase in F.I. in T2DM, ATH, and T2DM- 


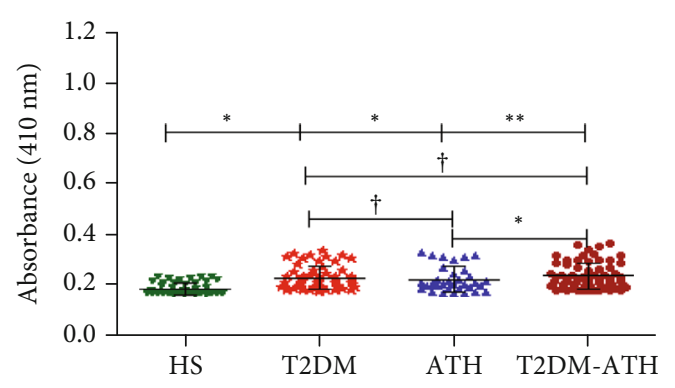

(a)

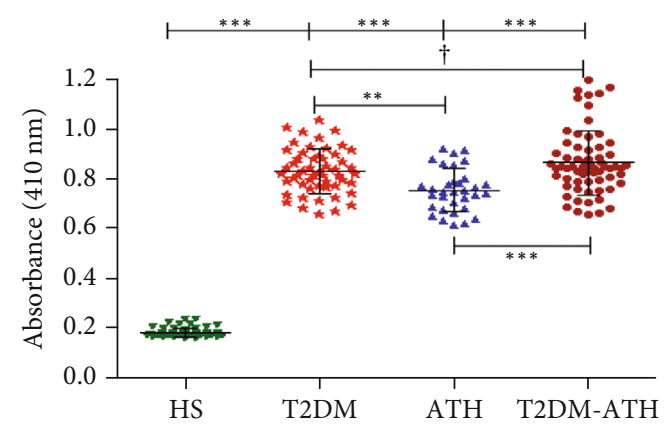

(b)

Figure 2: Direct binding ELISA of isolated IgG. (a) Direct binding ELISA of isolated IgG from diabetic (T2DM, $n=54)$, atherosclerosis (ATH, $n=33$ ), and diabetic atherosclerosis (T2DM-ATH, $n=65)$ patients with respect to healthy subjects (HS, $n=50)$ against N-Fib $\left(10 \mu \mathrm{g} \mathrm{mL} L^{-1}\right)$. All the three patient groups showed significant binding $\left({ }^{*} p<0.05\right.$ and $\left.{ }^{* *} p<0.001\right)$ with respect to HS. Data are presented as mean \pm SD of three independent experiments. (b) Direct binding ELISA of isolated IgG of diabetic (T2DM, $n=54)$, atherosclerosis (ATH, $n=33$ ), and diabetic atherosclerosis (T2DM-ATH, $n=65)$ patients with respect to healthy subjects (HS, $n=50)$ against MG-Fib $\left(10 \mu \mathrm{g} \mathrm{mL} L^{-1}\right)$. All the three patient groups showed a significant binding affinity with respect to HS and among themselves $\left({ }^{*} p<0.05\right.$, ${ }^{* *} p<0.001$, and $\left.{ }^{* * *} p<0.0001\right)$, except between T2DM and T2DM-ATH $\left({ }^{\dagger} p>0.05\right)$. Data are presented as mean \pm SD of three independent experiments.

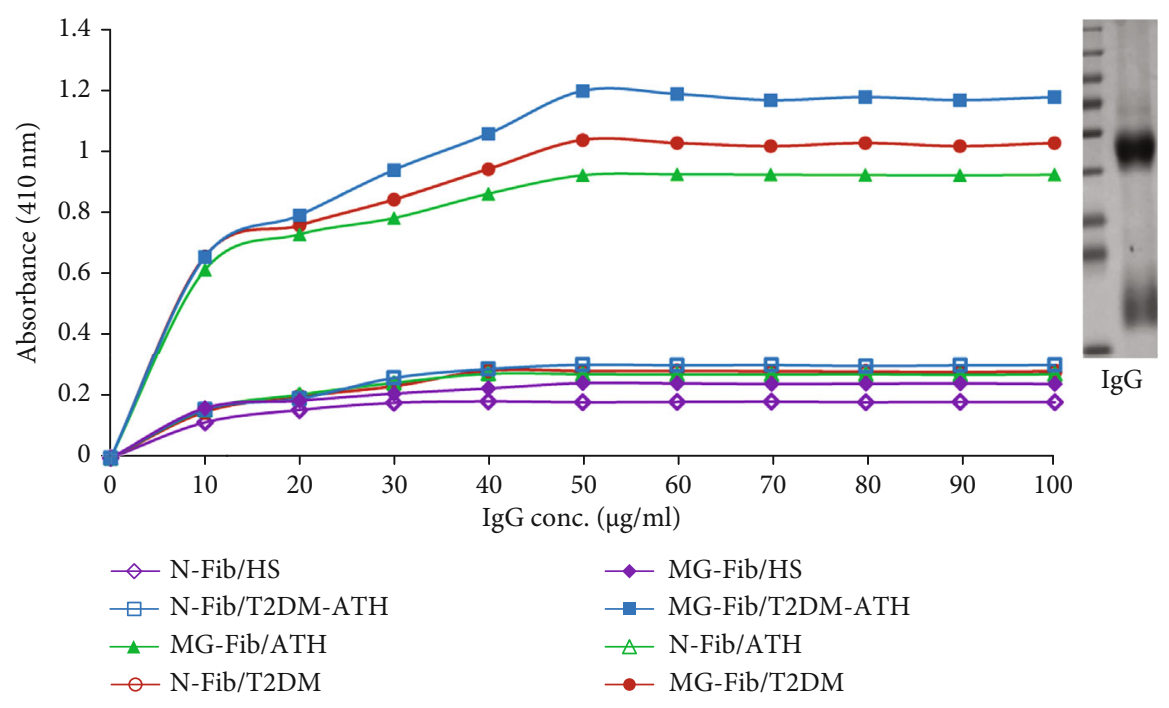

FIGURE 3: Binding affinity of purified IgG from diabetic (T2DM, $n=54)$, atherosclerosis (ATH, $n=33$ ), and diabetic atherosclerosis (T2DMATH, $n=65$ ) against native fibrinogen (N-Fib) and methylglyoxal- (MG-) glycated fibrinogen (MG-Fib). Insert: the image shows the marker and band (SDS PAGE) of IgG purified from patients' sera.

ATH groups with respect to the HS was $75 \%, 64 \%$, and $77 \%$, respectively (Figure 6). All the three patient groups exhibited statistical significance $(p<0.0001)$ with the HS group. The coefficient of variation in HS, T2DM, ATH, and T2DMATH groups was $29.81 \%, 18.84 \%, 16.19 \%$, and $17.91 \%$, respectively. The statistical data are summarized in Table 4.

3.9. Protein Carbonyl Estimation in Sera of HS and T2DM, $A T H$, and T2DM-ATH Patients. In the HS group, the mean concentration of protein-bound carbonyls was found to be $7.68 \pm 2.61 \mathrm{nM} \mathrm{mL}^{-1}$ (3.50-11.60), whereas in T2DM, ATH, and T2DM-ATH, the carbonyl contents were higher up to $34.79 \pm 8.59$ (20.64-52.05), $26.73 \pm 6.40$ (16.12-40.16), and $39.52 \pm 8.48 \mathrm{nM} \mathrm{mL}^{-1}(25.75-56.31)$, respectively. The percent increase in protein-bound carbonyls in T2DM, ATH, and T2DM-ATH groups with respect to HS was 77\%, 71\%, and $78 \%$, respectively (Figure 7 ). All the three patient groups exhibit statistical significance $(p<0.0001)$ with respect to the HS group. The coefficient of variation in HS, T2DM, ATH, and T2DM-ATH groups was 34.44\%, 24.63\%, 23.95\%, and $21.25 \%$, respectively. The statistical data are summarized in Table 4.

3.10. HMF Estimation in Sera of HS and T2DM, ATH, and T2DM-ATH Patients. In the HS group, the mean concentration of HMF was found to be $0.683 \pm 0.17 \mathrm{nM} \mathrm{mL}^{-1}(0.50-$ 1.14), whereas in T2DM, ATH, and T2DM-ATH, the HMF were higher up to $4.48 \pm 2.03(1.59-8.90), 2.40 \pm 1.01$ (1.29-4.51), and $6.02 \pm 2.67 \mathrm{nM} \mathrm{mL}^{-1}$ (2.10-11.21), respectively (Figure 8). The percent increase in HMF in T2DM, 


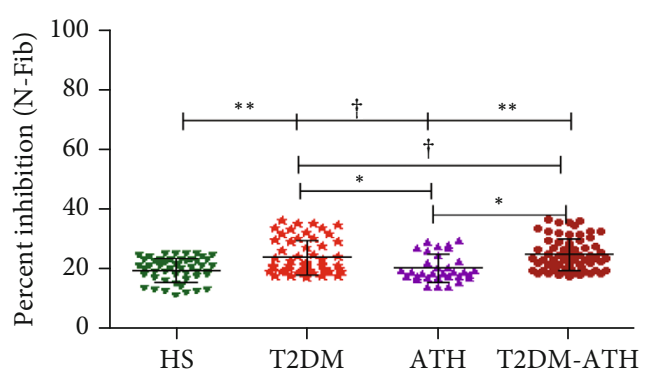

(a)

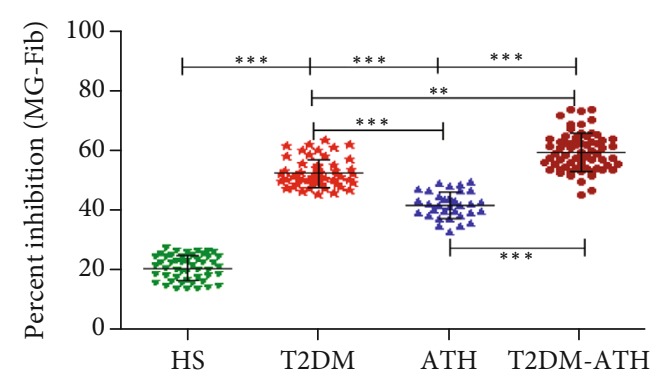

(b)

FIgure 4: Competitive ELISA. (a) Competitive ELISA of isolated IgG from healthy subjects (HS, $n=50$ ) and diabetic (T2DM, $n=54$ ), atherosclerosis $(\mathrm{ATH}, n=33)$, and diabetic atherosclerosis (T2DM-ATH, $n=65)$ patients with the native immune complex $(\mathrm{N}-\mathrm{Fib}+\mathrm{N}$ Fib-IgG). T2DM and T2DM-ATH groups show significant percent inhibition with respect to the HS group $\left({ }^{* *} p<0.001\right)$. The ATH group shows a nonsignificant $\left({ }^{\dagger} p>0.05\right)$ percent inhibition with respect to the HS group $\left({ }^{\dagger} p>0.05\right)$. Data are presented as mean \pm SD of three independent experiments. (b) Competitive ELISA of isolated IgG from healthy subjects (HS, $n=50)$ and diabetic (T2DM, $n=54$ ), atherosclerosis $(\mathrm{ATH}, n=33)$, and diabetic atherosclerosis (T2DM-ATH, $n=65)$ patients with the glycated immune complex (MG-Fib + MG-Fib-IgG). All the three patient groups showed significant percent inhibition with respect to the HS group and among themselves $\left({ }^{*} p<0.05,{ }^{* *} p<0.001\right.$, and $\left.{ }^{* * *} p<0.0001\right)$. Data are presented as mean \pm SD of three independent experiments.

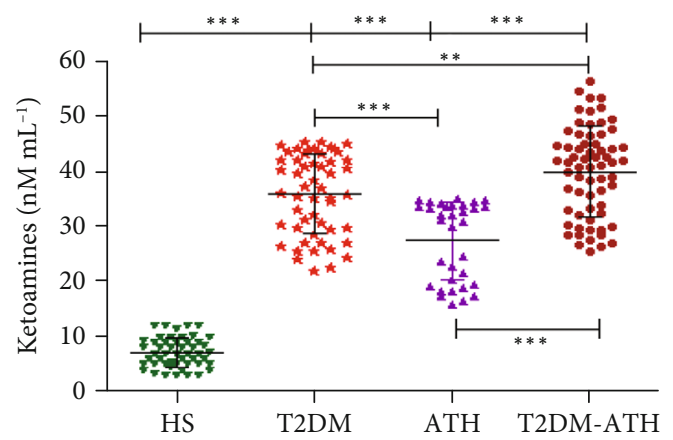

FIGURE 5: Ketoamine concentration $\left(\mathrm{nM} \mathrm{mL}^{-1}\right)$ in the sera of healthy subjects (HS, $n=50$ ) and diabetic (T2DM, $n=54$ ), atherosclerosis (ATH, $n=33$ ), and diabetic atherosclerosis (T2DM-ATH, $n=65$ ) patients. The HS group was used as a control. All the three patient groups showed statistical significance with respect to the HS group and among themselves $\left({ }^{* *} p<0.001,{ }^{* * *} p<0.0001\right)$. Data are presented as mean \pm SD of three independent experiments.

ATH, and T2DM-ATH groups with respect to healthy subjects was $84 \%, 71 \%$, and $88 \%$, respectively. All the three patient groups exhibit statistical significance $(p<0.0001)$ with the HS group. The coefficient of variation in HS, T2DM, ATH, and T2DM-ATH groups was $34.44 \%$, $45.23 \%, 42.10 \%$, and $44.39 \%$, respectively. The statistical data are summarized in Table 4.

3.11. TBARS Estimation in Sera of HS and T2DM, ATH, and T2DM-ATH Patients. In the HS group, the mean concentration of TBARS was found to be $3.94 \pm 0.42 \mu \mathrm{M} \mathrm{mL}^{-1}$ (3.154.65), whereas in T2DM, ATH, and T2DM-ATH, the TBARS levels were higher up to $30.94 \pm 5.16$ (22.17-38.54), $24.62 \pm 5.70 \quad(15.87-35.71), \quad$ and $34.42 \pm 6.63 \mu \mathrm{MmL}^{-1}$ (24.17-46.52), respectively (Figure 9). The percent increase in TBARS in T2DM, ATH, and T2DM-ATH groups with respect to healthy subjects was $87 \%, 83 \%$, and $88 \%$, respectively. All the three patient groups exhibit statistical signifi- cance $(p<0.0001)$ with the HS group. The coefficient of variation in $\mathrm{HS}, \mathrm{T} 2 \mathrm{DM}, \mathrm{ATH}$, and T2DM-ATH groups was $10.78 \%, 16.70 \%, 23.17 \%$, and $19.26 \%$, respectively. The statistical data are summarized in Table 4 .

3.12. Estimation of Free Lysine in HS and T2DM, ATH, and T2DM-ATH Patients. In the HS group, the mean absorbance at $420 \mathrm{~nm}$ was found to be $0.181 \pm 0.007(0.017-0.194)$, whereas in T2DM, ATH, and T2DM-ATH, the absorbance was lower up to $0.107 \pm 0.012(0.083-0.125), 0.116 \pm 0.010$ (0.100-0.139), and $0.088 \pm 0.014$ (0.061-0.130), respectively. The percent decrease in free lysine in T2DM, ATH, and T2DM-ATH groups with respect to healthy subjects was $40 \%, 35 \%$, and $51 \%$, respectively (Figure 10 ). All the three patient groups exhibit statistical significance $(p<0.0001)$ with the HS group. The coefficient of variation in HS, $\mathrm{T} 2 \mathrm{DM}, \mathrm{ATH}$, and T2DM-ATH groups was $4.07 \%, 12.08 \%$, $8.77 \%$, and $16.85 \%$, respectively. The statistical data are summarized in Table 4.

3.13. Estimation of Free Arginine in HS and T2DM, ATH, and T2DM-ATH Patients. In the HS group, the mean emission fluorescence intensity (F.I.) at $395 \mathrm{~nm}$ was found to be $102.91 \pm 8.18$ (a.u.) (90.17-120.33), whereas in T2DM, $\mathrm{ATH}$, and T2DM-ATH, the mean F.I. were lower up to $66.21 \pm 5.11$ (55.28-75.63), $74.44 \pm 7.39$ (60.12-86.75), and $61.39 \pm 11.54$ (a.u.) (44.09-84.91), respectively (Figure 11). The percent decrease in free arginine in T2DM, ATH, and T2DM-ATH groups with respect to healthy subjects was $35 \%, 27 \%$, and $40 \%$, respectively. All the three patient groups exhibit statistical significance $(p<0.0001)$ with respect to the HS group. The coefficient of variation in HS, T2DM, ATH, and T2DM-ATH groups was $7.95 \%, 7.72 \%$, $9.94 \%$, and $18.71 \%$, respectively. The statistical data are summarized in Table 4.

3.14. CML Estimation in Sera of HS and T2DM, ATH, and T2DM-ATH Patients. In the HS group, the mean concentration of CML was found to be $0.215 \pm 0.026 \mathrm{nM} \mathrm{mL}^{-1}(0.170$ - 
TABLE 4: Statistical data showing ketoamines, AGEs, protein carbonyls, hydroxymethyl furfural (HMF), TBARS, free lysine, free arginine, and carboxymethyllysine concentrations in the sera of HS, T2DM, ATH, and T2DM-ATH patient groups.

\begin{tabular}{|c|c|c|c|c|c|c|c|c|}
\hline Disease & $N$ & Mean & SD & Min. & Max. & $\mathrm{CV}$ & $p$ value & $\%$ Inc/Dec \\
\hline \multicolumn{9}{|l|}{ Ketoamines } \\
\hline HS & 50 & 7.15 & 2.65 & 3.12 & 12.43 & $37.06 \%$ & - & \\
\hline T2DM & 54 & 36.03 & 7.30 & 21.98 & 45.32 & $20.28 \%$ & $p<0.0001$ & $80 \% \uparrow$ \\
\hline ATH & 33 & 27.52 & 7.16 & 16.04 & 35.19 & $26.04 \%$ & $p<0.0001$ & $72 \% \uparrow$ \\
\hline T2DM-ATH & 65 & 39.83 & 7.86 & 25.51 & 52.62 & $19.73 \%$ & $p<0.0001$ & $82 \% \uparrow$ \\
\hline \multicolumn{9}{|l|}{ AGEs } \\
\hline HS & 50 & 16.03 & 4.78 & 9.34 & 25.47 & $29.81 \%$ & - & \\
\hline T2DM & 54 & 65.37 & 12.31 & 47.28 & 83.54 & $18.84 \%$ & $p<0.0001$ & $75 \% \uparrow$ \\
\hline ATH & 33 & 45.47 & 7.36 & 31.23 & 60.49 & $16.19 \%$ & $p<0.0001$ & $64 \% \uparrow$ \\
\hline T2DM-ATH & 65 & 71.90 & 13.13 & 42.21 & 88.61 & $17.91 \%$ & $p<0.0001$ & $77 \% \uparrow$ \\
\hline \multicolumn{9}{|c|}{ Carbonyl content } \\
\hline HS & 50 & 7.68 & 2.61 & 3.50 & 11.60 & $34.44 \%$ & - & \\
\hline T2DM & 54 & 34.79 & 8.56 & 20.64 & 52.05 & $24.63 \%$ & $p<0.0001$ & $77 \% \uparrow$ \\
\hline ATH & 33 & 26.73 & 6.40 & 16.12 & 40.16 & $23.95 \%$ & $p<0.0001$ & $71 \% \uparrow$ \\
\hline T2DM-ATH & 65 & 39.52 & 8.48 & 25.75 & 56.31 & $21.25 \%$ & $p<0.0001$ & $78 \% \uparrow$ \\
\hline \multicolumn{9}{|c|}{ Hydroxymethyl furfural } \\
\hline HS & 50 & 0.68 & 0.17 & 0.50 & 1.14 & $25.08 \%$ & - & \\
\hline $\mathrm{T} 2 \mathrm{DM}$ & 54 & 4.48 & 2.03 & 1.59 & 8.90 & $45.23 \%$ & $p<0.0001$ & $84 \% \uparrow$ \\
\hline ATH & 33 & 2.40 & 1.01 & 1.29 & 4.51 & $42.10 \%$ & $p<0.0001$ & $71 \% \uparrow$ \\
\hline T2DM-ATH & 65 & 6.02 & 2.67 & 2.10 & 11.21 & $44.39 \%$ & $p<0.0001$ & $88 \% \uparrow$ \\
\hline \multicolumn{9}{|l|}{ TBARS } \\
\hline HS & 50 & 3.94 & 0.42 & 3.15 & 4.65 & $10.78 \%$ & - & \\
\hline T2DM & 54 & 30.94 & 5.16 & 22.17 & 38.54 & $16.70 \%$ & $p<0.0001$ & $87 \% \uparrow$ \\
\hline ATH & 33 & 24.62 & 5.70 & 15.87 & 35.71 & $23.17 \%$ & $p<0.0001$ & $83 \% \uparrow$ \\
\hline T2DM-ATH & 65 & 34.42 & 6.63 & 24.17 & 46.52 & $19.26 \%$ & $p<0.0001$ & $88 \% \uparrow$ \\
\hline \multicolumn{9}{|l|}{ Free lysine } \\
\hline HS & 50 & 0.181 & 0.007 & 0.171 & 0.194 & $4.07 \%$ & - & \\
\hline T2DM & 54 & 0.107 & 0.012 & 0.083 & 0.125 & $12.08 \%$ & $p<0.0001$ & $40 \% \downarrow$ \\
\hline ATH & 33 & 0.116 & 0.010 & 0.100 & 0.139 & $8.77 \%$ & $p<0.0001$ & $35 \% \downarrow$ \\
\hline T2DM-ATH & 65 & 0.088 & 0.014 & 0.061 & 0.130 & $16.85 \%$ & $p<0.0001$ & $51 \% \downarrow$ \\
\hline \multicolumn{9}{|l|}{ Free arginine } \\
\hline HS & 50 & 102.91 & 8.18 & 90.17 & 120.33 & $7.95 \%$ & - & \\
\hline $\mathrm{T} 2 \mathrm{DM}$ & 54 & 66.21 & 5.11 & 55.28 & 75.63 & $7.72 \%$ & $p<0.0001$ & $35 \% \downarrow$ \\
\hline ATH & 33 & 74.44 & 7.39 & 60.12 & 86.75 & $9.94 \%$ & $p<0.0001$ & $27 \% \downarrow$ \\
\hline T2DM-ATH & 65 & 61.39 & 11.54 & 44.09 & 84.91 & $18.17 \%$ & $p<0.0001$ & $40 \% \downarrow$ \\
\hline \multicolumn{9}{|c|}{ Carboxymethyllysine } \\
\hline HS & 50 & 0.215 & 0.026 & 0.170 & 0.257 & $12.31 \%$ & - & \\
\hline $\mathrm{T} 2 \mathrm{DM}$ & 54 & 0.814 & 0.141 & 0.654 & 1.159 & $17.36 \%$ & $p<0.0001$ & $73 \% \uparrow$ \\
\hline $\mathrm{ATH}$ & 33 & 0.653 & 0.084 & 0.524 & 0.821 & $12.95 \%$ & $p<0.0001$ & $67 \% \uparrow$ \\
\hline T2DM-ATH & 65 & 0.889 & 0.186 & 0.653 & 1.447 & $21.34 \%$ & $p<0.0001$ & $75 \% \uparrow$ \\
\hline
\end{tabular}

The data was presented as mean $\pm \mathrm{SD}$, and the statistical significance level $(p<0.05)$ between HS, T2DM, ATH, and T2DM-ATH groups was determined by one-way ANOVA. All the three patient groups exhibit statistical significance $(p<0.0001)$ with the HS group. " $N$ " is the no. of subjects in each group, "Min." is the minimum value, "Max." is the maximum value, "SD" is the standard deviation, "CV" is the coefficient of variation, and " $p$ " is the level of significance. The upward arrows $(\uparrow)$ represent percent increase whereas downward arrows $(\downarrow)$ represent percent decrease in parameters with respect to the HS group. 


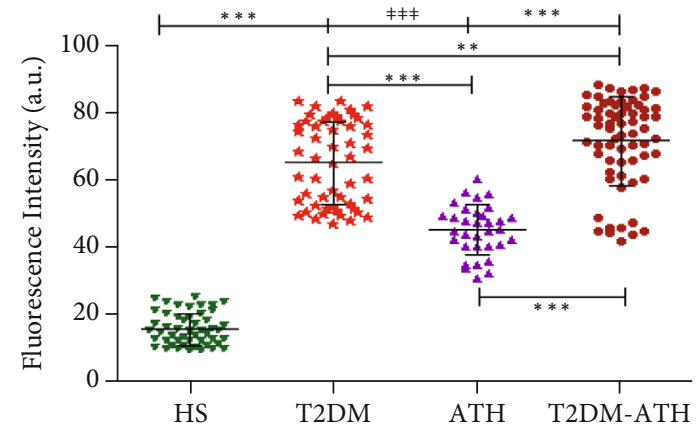

FIGURE 6: Fluorescence emission profiles at $460 \mathrm{~nm}$ upon excitation at $370 \mathrm{~nm}$ for the detection of fluorescent AGEs in the sera of healthy subjects (HS, $n=50$ ) and diabetic (T2DM, $n=54$ ), atherosclerosis (ATH, $n=33$ ), and diabetic atherosclerosis (T2DM-ATH, $n=65$ ) patients. The HS group was used as a control. All the three patient groups showed statistical significance with respect to the HS group and among themselves $\left({ }^{* *} p<0.001,{ }^{* * *} p<0.0001\right)$. Data are presented as mean $\pm \mathrm{SD}$ of three independent experiments.

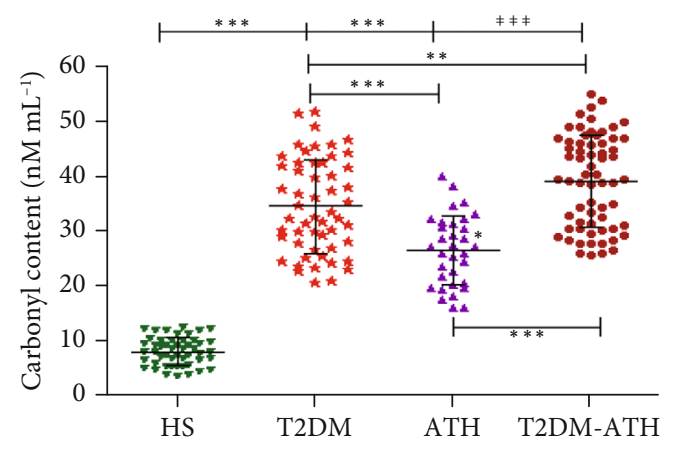

FIGURE 7: Protein carbonyl concentration $\left(\mathrm{nM} \mathrm{mL}^{-1}\right)$ in the sera of healthy subjects (HS, $n=50$ ) and diabetic (T2DM, $n=54$ ), atherosclerosis (ATH, $n=33$ ), and diabetic atherosclerosis (T2DM-ATH, $n=65$ ) patients. The HS group was used as a control. All the three patient groups showed statistical significance with respect to the HS group and among themselves $\left({ }^{* *} p<0.001,{ }^{* * *} p<0.0001\right)$. Data are presented as mean $\pm S D$ of three independent experiments.

0.257), whereas in T2DM, ATH, and T2DM-ATH, the CML were higher up to $0.814 \pm 0.141(0.654-1.159), 0.653 \pm 0.084$ $(0.524-0.821)$, and $0.889 \pm 0.186 \mathrm{nM} \mathrm{mL}^{-1}(0.653-1.447)$, respectively (Figure 12). The percent increase in CML concentration in T2DM, ATH, and T2DM-ATH groups with respect to healthy subjects was $73 \%, 67 \%$, and $75 \%$, respectively. All the three patient groups exhibit statistical significance $(p<0.0001)$ with respect to the HS group. The coefficient of variation in HS, T2DM, ATH, and T2DMATH groups was $12.31 \%, 17.36 \%, 12.95 \%$, and $21.34 \%$, respectively. The statistical data are summarized in Table 4.

\section{Discussion}

Reducing sugars along with dicarbonyls or reactive carbonyl species (RCS) such as methylglyoxal (MG) can instigate

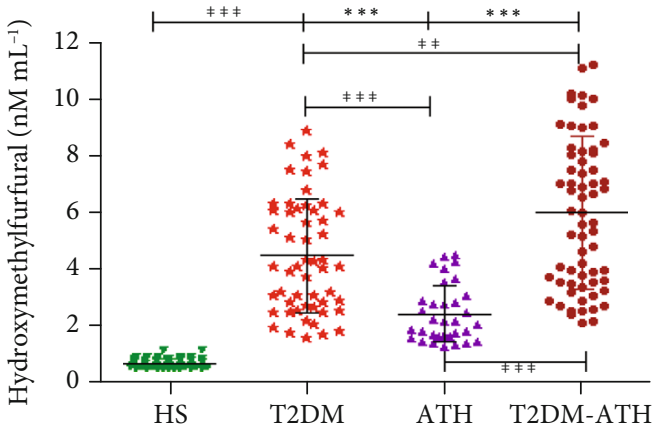

Figure 8: Hydroxymethylfurfural concentration $\left(\mathrm{nM} \mathrm{mL}^{-1}\right)$ in the sera of healthy subjects (HS, $n=50$ ) and diabetic (T2DM, $n=54$ ), atherosclerosis (ATH, $n=33$ ), and diabetic atherosclerosis (T2DM-ATH, $n=65$ ) patients. The HS group was used as a control. All the three patient groups showed statistical significance with respect to the HS group and among themselves $\left({ }^{* *} p<0.001,{ }^{* * *} p<0.0001\right)$. Data are presented as mean \pm SD of three independent experiments.

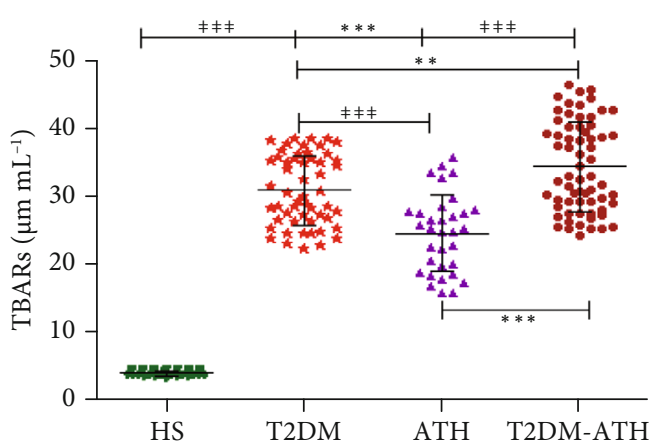

FIGURE 9: TBARS concentration $\left(\mu \mathrm{M} \mathrm{mL}^{-1}\right)$ in the sera of healthy subjects (HS, $n=50)$ and diabetic (T2DM, $n=54)$, atherosclerosis (ATH, $n=33$ ), and diabetic atherosclerosis (T2DM-ATH, $n=65$ ) patients. The HS group was used as a control. All the three patient groups showed statistical significance with respect to the HS group and among themselves $\left({ }^{* *} p<0.001,{ }^{* * *} p<0.0001\right)$. Data are presented as mean \pm SD of three independent experiments.

in vivo nonenzymatic glycosylation [18]. This reaction is a slow process but increases several folds during prolonged and persistent hyperglycemia [4]. It has been investigated that MG is a highly reactive dicarbonyl that leads to cellular dysfunction by perturbing native structure and normal function of proteins. Moreover, in the persistent hyperglycemic state, the concentration of dicarbonyls was significantly elevated [11]. Furthermore, exposure and generation of neoepitopes on glycated proteins render them highly immunogenic which leads to the production of autoantibodies [44].

The direct binding ELISA of patients' sera has revealed a higher prevalence of autoantibodies in type 2 diabetes mellitus (T2DM, 54\%), atherosclerosis (ATH, 33\%), and diabetic atherosclerosis (T2DM-ATH, 65\%) against MG-Fib. However, healthy subject (HS) sera did not show significant binding with both the N-Fib and MG-Fib. From our in vitro characterization study, it was implied that MG-mediated 


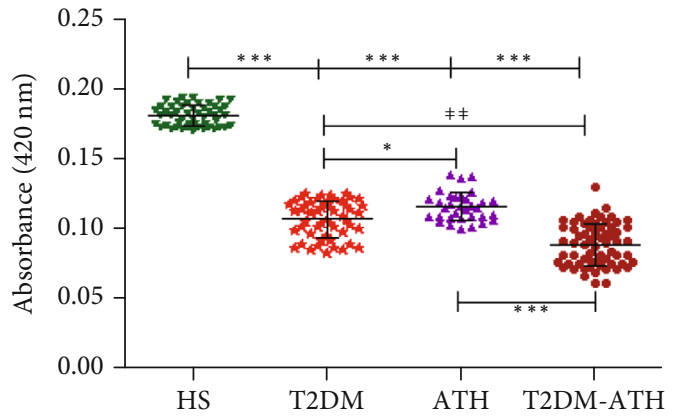

FIGURE 10: Absorbance profiles of free lysine residues in the sera of healthy subjects (HS, $n=50$ ) and diabetic (T2DM, $n=54$ ), atherosclerosis (ATH, $n=33$ ), and diabetic atherosclerosis (T2DM-ATH, $n=65$ ) patients. The HS group was used as a control. All the three patient groups showed statistical significance with respect to the HS group and among themselves $\left({ }^{*} p<0.05,{ }^{* *} p<0.001\right.$, and $\left.{ }^{* * *} p<0.0001\right)$. Data are presented as mean \pm SD of three independent experiments.

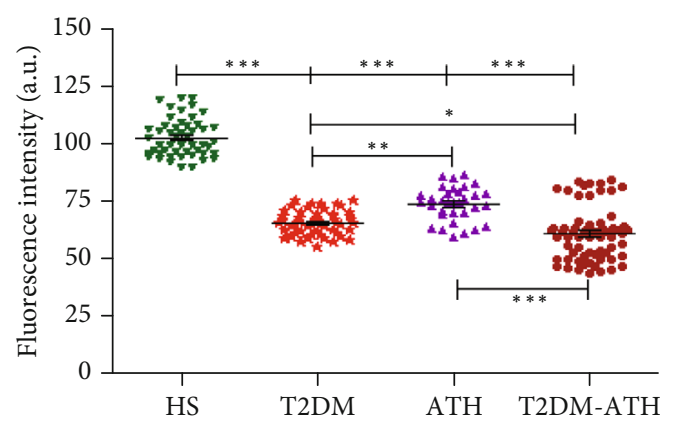

Figure 11: Fluorescence emission profiles at $395 \mathrm{~nm}$ upon excitation at $280 \mathrm{~nm}$ for the detection of free arginine residues in the sera of healthy subjects (HS, $n=50)$ and diabetic (T2DM, $n=$ $54)$, atherosclerosis (ATH, $n=33$ ), and diabetic atherosclerosis (T2DM-ATH, $n=65$ ) patients. The HS group was used as a control. All the three patient groups showed statistical significance with respect to the HS group and among themselves $\left({ }^{*} p<0.05,{ }^{* *} p<0.001\right.$, and $\left.{ }^{* * *} p<0.0001\right)$. Data are presented as mean \pm SD of three independent experiments.

glycation of fibrinogen leads to the formation of fibrinogenAGEs (Fib-AGEs), which might elicit immune response via neoepitope generation [38]. The presence of highly specific anti-MG-Fib autoantibodies or anti-Fib-AGEs autoantibodies in the sera of T2DM (54\%), ATH (33\%), and T2DM-ATH (65\%) patients is in agreement with our in vitro study. Moreover, direct binding ELISA of sera against N-Fib also showed significant binding in some samples of T2DM and T2DM-ATH patient groups. The reason might be the denaturation and exposure of neoepitopes on $\mathrm{N}$-Fib during incubation at $37^{\circ} \mathrm{C}$ for 7 days.

The direct binding of isolated IgG from those patients' sera that showed higher affinity towards MG-Fib further validates the generation of autoantibodies (anti-MG-FibIgG) in the patient groups. Moreover, autoantibodies (anti-N-Fib-IgG) against N-Fib were also found in all the three patient groups and showed significant binding with

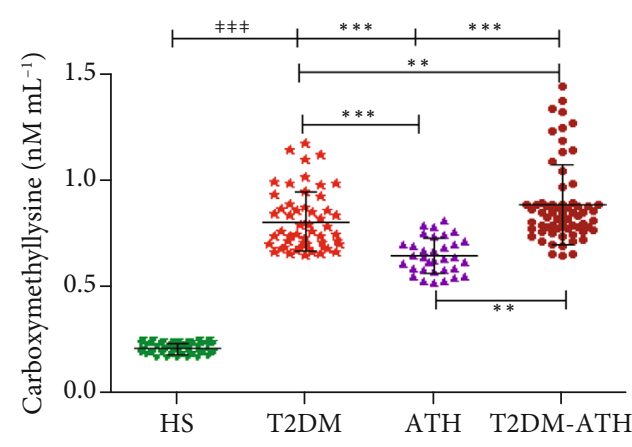

FIGURE 12: Carboxymethyllysine concentration $\left(\mathrm{nM} \mathrm{mL}^{-1}\right)$ in the sera of healthy subjects (HS, $n=50$ ) and diabetic (T2DM, $n=54$ ), atherosclerosis (ATH, $n=33$ ), and diabetic atherosclerosis (T2DM-ATH, $n=65$ ) patients. The HS group was used as a control. All the three patient groups showed statistical significance with respect to the HS group and among themselves $\left({ }^{*} p<0.05,{ }^{* *} p<0.001\right.$, and $\left.{ }^{* * *} p<0.0001\right)$. Data are presented as mean \pm SD of three independent experiments.

respect to the HS group. However, the absorbance at $410 \mathrm{~nm}$ was much lower in N-Fib with respect to MG-Fib. The results are in agreement with direct binding ELISA of sera against $\mathrm{N}-\mathrm{Fib}$.

In competitive/inhibition ELISA, the maximum inhibition of isolated IgG against N-Fib and MG-Fib follows the same descending order: T2DM $-\mathrm{ATH}>\mathrm{T} 2 \mathrm{DM}>\mathrm{ATH}$. However, in the case of N-Fib, the maximum percent inhibition among T2DM and T2DM-ATH patient groups with respect to the HS group was found to be significant $(p<0.05)$, whereas maximum percent inhibition against MG-Fib in the patient groups with respect to the HS group was significant $(p<0.05)$. Thus, the results suggest that the high specificity of serum autoantibodies or isolated IgG towards MG-Fib is due to the generation of neoepitopes on MG-modified fibrinogen as compared to N-Fib. Moreover, percent inhibition towards N-Fib reveals that N-Fib gets denatured during incubation and neoepitopes are exposed, against which autoantibodies (anti-N-Fib-IgG) are generated.

The NBT reduction assay of patients' sera suggests a 5-6fold increase in ketoamine concentration in T2DM and T2DM-ATH patients with respect to healthy subjects. However, in ATH patients, 3-fold increase in ketoamines was noticed in comparison to that in healthy individuals. The early glycation products reduce the yellow NBT to purple monoformazan. It has been suggested that NBT is reduced by the action of superoxide radicals that are formed during the degradation of ketoamines [61, 62]. Furthermore, the superoxide radicals via the Fenton reaction give rise to hydroxyl radicals. Thus, it is hypothesized that both superoxide and hydroxyl radicals might cause damage to protein molecules besides glycation $[63,64]$.

The fluorescence emission profiles at $460 \mathrm{~nm}$ confirmed the presence of AGEs in T2DM, ATH, and T2DM-ATH patients' sera. In comparison to healthy individuals, the patient groups exhibit a 2-4-fold increase in fluorescence intensities (F.I.). However, in the ATH group, there is a $\sim 10 \%$ decline in F.I. with respect to T2DM and T2DM-ATH 
groups which indicates more generation of fluorescent AGEs under a hyperglycemic state. Moreover, it has been reported that the fluorescence is exhibited only by those AGEs that have heterocyclic structures $[65,66]$.

Protein carbonyls are the markers of oxidative stress formed via enediol reaction from ketoamines $(67,68)$. During enediol reaction, superoxide radicals are formed that give rise to hydroxyl radicals via the Fenton reaction that further contributes to oxidative stress $(69,70)$. In our study, 3-5-fold increase in carbonyl contents in T2DM, ATH, and T2DM-ATH patients confirms higher oxidative stress. However, in the ATH group, the carbonyl contents were lower in concentration than those in T2DM and T2DM-ATH further authenticating hyperglycemia-induced oxidative stress in diabetic subjects. Earlier studies on diabetic patients suggest that increased accumulation of carbonyl contents exerts an adverse effect on physiological systems $(71,72)$. Both hydroxymethylfurfural (HMF) and malondialdehyde (MDA) are thiobarbituric acid (TBA) reactive species (TBARS) (38). $\mathrm{HMF}$ is released upon hydrolysis of ketoamines by weak acids, whereas MDA is an indicator of oxidative stress generated during oxidation of proteins to protein carbonyls via enediol reaction $(60,73)$. In our study, we found a 3-8-fold increase in HMF content and a 6-8-fold increase in MDA concentration in T2DM, ATH, and T2DM-ATH patients with respect to healthy subjects. However, in the ATH group, the HMF contents and MDA concentration were lesser than those in T2DM and T2DM-ATH. This indicates that more oxidative stress is generated during diabetesinduced prolonged and persistent hyperglycemia. The results indicate enhanced oxidative stress in diabetic and diabetes atherosclerosis patients due to hyperglycemiamediated accelerated protein glycation. The ketoamines, carbonyl contents, HMF, and TBARS results are in agreement with each other.

The lysine (Lys) and arginine (Arg) amino acids are more susceptible to glycation because of the presence of free amino group $\left(-\mathrm{NH}_{2}\right)$ and guanidino group in their respective side chains (59). In our study, the significant drop in free Lys and free Arg in T2DM and T2DM-ATH patients with respect to the HS group portrays the extent of glycation and AGE formation. The percentage of reacted lysine and arginine residues in T2DM and T2DM-ATH patients is much higher with respect to healthy individuals.

CML is a nonfluorogenic AGE formed in hyperglycemic individuals (74-76). In our study, a 3-4-fold increase in CML levels in the patient groups suggests increased glycative stress in T2DM and T2DM-ATH patients in comparison to healthy individuals.

\section{Conclusion}

The present study explored methylglyoxal-mediated structural perturbations induced in fibrinogen protein that results in the generation of neoepitopes, which in turn provokes an immune response in the form of autoantibodies (anti-MGFib antibodies) in T2DM, ATH, and T2DM-ATH patients. However, the precise role of glycated fibrinogen in the pathogenesis of diabetes-associated micro- and macrovascular complications is still in veil and needs further investigation. We hope that our findings may provide an approach to predict diabetes-related atherosclerosis before its onset by validating glycated fibrinogen as a new biomarker. Moreover, this study might facilitate the scientific fraternity to minimize the menace of glycation-induced oxidative and carbonyl stress, by inhibiting and scavenging the intermediates of nonenzymatic glycosylation.

$\begin{array}{ll}\text { Abbreviations } & \\ \text { MG: } & \text { Methylglyoxal } \\ \text { Fib: } & \text { Fibrinogen } \\ \text { N-Fib: } & \text { Native fibrinogen } \\ \text { MG-Fib: } & \begin{array}{l}\text { MG-glycated fibrinogen/or MG- } \\ \text { modified fibrinogen }\end{array} \\ \text { N-Fib-IgG: } & \text { Anti-N-Fib autoantibodies } \\ \text { MG-Fib-IgG: } & \text { Anti-MG-fib autoantibodies } \\ \text { N-Fib+N-Fib-IgG: } & \text { Native immune complex } \\ \text { MG-Fib+MG-Fib- } & \text { Glycated immune complex } \\ \text { IgG: } & \\ \text { HS: } & \text { Healthy subjects } \\ \text { T2DM: } & \text { Type 2 diabetes mellitus } \\ \text { ATH: } & \text { Atherosclerosis } \\ \text { T2DM-ATH: } & \text { Diabetic atherosclerosis. }\end{array}$

\section{Data Availability}

Data is available within the text of the manuscript.

\section{Conflicts of Interest}

The authors declare that they have no conflict of interest.

\section{Authors' Contributions}

Shahnawaz Rehman and Saheem Ahmad contributed equally to this manuscript, so both are considered the first authors.

\section{Acknowledgments}

This study was supported by the Researchers Supporting Project (RSP-2021/86), King Saud University, Riyadh, Saudi Arabia, and Outstanding Innovation Team Promotion Plan of Shandong Provincial Hospital Affiliated to Shandong First Medical University.

\section{References}

[1] M. Brownlee, "The pathobiology of diabetic complications: a unifying mechanism," Diabetes, vol. 54, no. 6, pp. 16151625, 2005.

[2] V. L. Bodiga, S. R. Eda, and S. Bodiga, "Advanced glycation end products: role in pathology of diabetic cardiomyopathy," Heart Failure Reviews, vol. 19, no. 1, pp. 49-63, 2014.

[3] H. Sáenz-Suárez, R. A. POUTOU-Piñales, J. González-Santos et al., "Prediction of glycation sites: new insights from protein structural analysis," Turkish Journal of Biology, vol. 40, no. 1, pp. 12-25, 2016. 
[4] S. Ahmad, Z. Siddiqui, S. Rehman et al., "A glycation angle to look into the diabetic vasculopathy: cause and cure," Current Vascular Pharmacology, vol. 15, no. 4, pp. 352-364, 2017.

[5] J. D. Méndez, J. Xie, M. Aguilar-Hernández, and V. MéndezValenzuela, "Molecular susceptibility to glycation and its implication in diabetes mellitus and related diseases," Molecular and Cellular Biochemistry, vol. 344, no. 1-2, pp. 185-193, 2010.

[6] S. Ahmad, M. Y. Khan, Z. Rafi et al., "Oxidation, glycation and glycoxidation-The vicious cycle and lung cancer," in Seminars in Cancer Biology, vol. 49, pp. 29-36, Academic Press, 2018.

[7] P. Newsholme, V. F. Cruzat, K. N. Keane, R. Carlessi, and P. I. H. de Bittencourt, "Molecular mechanisms of ROS production and oxidative stress in diabetes," Biochemical Journal, vol. 473, no. 24, pp. 4527-4550, 2016.

[8] L. N. Bavkar, R. S. Patil, S. B. Rooge, M. L. Nalawade, and A. U. Arvindekar, "Acceleration of protein glycation by oxidative stress and comparative role of antioxidant and protein glycation inhibitor," Molecular and Cellular Biochemistry, vol. 459, no. 1-2, pp. 61-71, 2019.

[9] H. M. Semchyshyn, "Reactive carbonyl species in vivo: generation and dual biological effects," The Scientific World Journal, vol. 2014, Article ID 417842, 10 pages, 2014.

[10] S. Brings, T. Fleming, M. Freichel, M. U. Muckenthaler, S. Herzig, and P. P. Nawroth, "Dicarbonyls and advanced glycation end-products in the development of diabetic complications and targets for intervention," International Journal of Molecular Sciences, vol. 18, no. 5, p. 984, 2017.

[11] P. J. Thornalley, A. Langborg, and H. S. Minhas, "Formation of glyoxal, methylglyoxal and 3-deoxyglucosone in the glycation of proteins by glucose," Biochemical Journal, vol. 344 , no. 1 , pp. 109-116, 1999.

[12] S. Ahmad, H. Khan, U. Shahab et al., "Protein oxidation: an overview of metabolism of sulphur containing amino acid, cysteine," Frontiers in Bioscience (Scholar Edition), vol. 9, no. 1, pp. 71-87, 2017.

[13] P. J. Thornalley and N. Rabbani, "Assay of methylglyoxal and glyoxal and control of peroxidase interference," Biochemical Society Transactions, vol. 42, no. 2, pp. 504-510, 2014.

[14] N. Rabbani and P. J. Thornalley, "Dicarbonyl proteome and genome damage in metabolic and vascular disease," Biochemical Society Transactions, vol. 42, no. 2, pp. 425-432, 2014.

[15] K. J. Rodnick, R. W. Holman, P. S. Ropski, M. Huang, and A. L. Swislocki, "A perspective on reagent diversity and noncovalent binding of reactive carbonyl species (RCS) and effector reagents in non-enzymatic glycation (NEG): mechanistic considerations and implications for future research," Frontiers in Chemistry, vol. 5, p. 39, 2017.

[16] T. J. Lyons, "Glycation and oxidation: a role in the pathogenesis of atherosclerosis," The American Journal of Cardiology, vol. 71, no. 6, pp. B26-B31, 1993.

[17] S. Ahmad, M. S. Khan, F. Akhter et al., "Glycoxidation of biological macromolecules: a critical approach to halt the menace of glycation," Glycobiology, vol. 24, no. 11, pp. 979-990, 2014.

[18] S. Ahmad, H. Khan, Z. Siddiqui et al., "AGEs, RAGEs and sRAGE; friend or foe for cancer," in Seminars in cancer biology, vol. 49, pp. 44-55, Academic Press, 2018.

[19] G. A. Tennent, S. O. Brennan, A. J. Stangou, J. O'Grady, P. N. Hawkins, and M. B. Pepys, "Human plasma fibrinogen is synthesized in the liver," Blood, vol. 109, no. 5, pp. 1971-1974, 2007.
[20] J. Zhang, Y. Wang, R. Zhang et al., "Serum fibrinogen predicts diabetic ESRD in patients with type 2 diabetes mellitus," Diabetes Research and Clinical Practice, vol. 141, pp. 1-9, 2018.

[21] M. Pieters, D. G. van Zyl, P. Rheeder et al., "Glycation of fibrinogen in uncontrolled diabetic patients and the effects of glycaemic control on fibrinogen glycation," Thrombosis Research, vol. 120, no. 3, pp. 439-446, 2007.

[22] C. P. Domingueti, L. M. S.'. A. Dusse, M. . G. Carvalho, L. P. de Sousa, K. B. Gomes, and A. P. Fernandes, "Diabetes mellitus: the linkage between oxidative stress, inflammation, hypercoagulability and vascular complications," Journal of Diabetes and its Complications, vol. 30, no. 4, pp. 738-745, 2016.

[23] A. Di Pino and R. A. DeFronzo, "Insulin resistance and atherosclerosis: implications for insulin-sensitizing agents," Endocrine Reviews, vol. 40, no. 6, pp. 1447-1467, 2019.

[24] M. W. Hatton, S. Southward, B. Ross-Ouellet, M. Richardson, and P. D. Winocour, "Catabolism of unglycated and naturally glycated forms of rabbit fibrinogen: their interaction with the healthy and deendothelialized aorta wall in normal and diabetic rabbits," The Journal of Laboratory and Clinical Medicine, vol. 121, no. 4, pp. 588-597, 1993.

[25] E. J. Dunn and R. A. Ariëns, "Fibrinogen and fibrin clot structure in diabetes," Herz, vol. 29, no. 5, pp. 470-479, 2004.

[26] E. J. Dunn, R. A. S. Ariëns, and P. J. Grant, "The influence of type 2 diabetes on fibrin structure and function," Diabetologia, vol. 48, no. 6, pp. 1198-1206, 2005.

[27] A. L. C. La Cote, H. Philippou, and R. A. Ariens, "Role of fibrin structure in thrombosis and vascular disease," in Advances in protein chemistry and structural biology, vol. 83, pp. 75-127, Academic Press, 2011.

[28] D. C. Rijken and S. Uitte de Willige, "Inhibition of fibrinolysis by coagulation factor XIII," BioMed research international, vol. 2017, Article ID 1209676, 6 pages, 2017.

[29] P. W. Robinson, D. R. Jury, and A. G. Langdon, “The relative clotting activity of glycated and non-glycated forms of fibrinogen," Annals of Clinical Biochemistry, vol. 28, no. 6, pp. 618619, 1991.

[30] M. Brownlee, H. Vlassara, and A. Cerami, "Nonenzymatic glycosylation reduces the susceptibility of fibrin to degradation by plasmin," Diabetes, vol. 32, no. 7, pp. 680-684, 1983.

[31] I. W. Bobbink, W. L. Tekelenburg, J. J. Sixma, H. C. de Boer, J. D. Banga, and P. G. de Groot, "Glycated Proteins Modulate Tissue-Plasminogen Activator-Catalyzed Plasminogen Activation," Biochemical and Biophysical Research Communications, vol. 240, no. 3, pp. 595-601, 1997.

[32] T. T. Marar, Regulation of the Activation and Function of Thrombin-Activatable Fibrinolysis Inhibitor (TAFI), 2016.

[33] R. Krogh-Madsen, P. Plomgaard, P. Keller, C. Keller, and B. K. Pedersen, "Insulin stimulates interleukin- 6 and tumor necrosis factor- $\alpha$ gene expression in human subcutaneous adipose tissue," American Journal of Physiology-Endocrinology and Metabolism, vol. 286, no. 2, pp. E234-E238, 2004.

[34] M. Soop, H. Duxbury, A. O. Agwunobi et al., "Euglycemic hyperinsulinemia augments the cytokine and endocrine responses to endotoxin in humans," American Journal of Physiology-Endocrinology and Metabolism, vol. 282, no. 6, pp. E1276-E1285, 2002.

[35] Y. Jinnouchi, H. Sano, R. Nagai et al., "Glycolaldehyde-modified low density lipoprotein leads macrophages to foam cells via the macrophage scavenger receptor," The Journal of Biochemistry, vol. 123, no. 6, pp. 1208-1217, 1998. 
[36] J. Y. Chen, Z. X. Ye, X. F. Wang et al., "Nitric oxide bioavailability dysfunction involves in atherosclerosis," Biomedicine \& Pharmacotherapy, vol. 97, pp. 423-428, 2018.

[37] R. Rabieian, M. Boshtam, M. Zareei, S. Kouhpayeh, A. Masoudifar, and H. Mirzaei, "Plasminogen activator inhibitor type-1 as a regulator of fibrosis," Journal of Cellular Biochemistry, vol. 119, no. 1, pp. 17-27, 2018.

[38] S. Rehman, M. Faisal, A. A. Alatar, and S. Ahmad, "Physicochemical changes induced in serum proteins, immunoglobulin-G and fibrinogen by reactive carbonyl species, methylglyoxal," Current Protein \& Peptide Science, vol. 20, pp. 1-10, 2019.

[39] S. Ahmad, M. Uddin, S. Habib, U. Shahab, K. Alam, and A. Ali, "Autoimmune response to AGE modified human DNA: implications in type 1 diabetes mellitus," Journal of Clinical \& Translational Endocrinology, vol. 1, no. 3, pp. 66-72, 2014.

[40] F. Akhter, M. S. Khan, and S. Ahmad, “Acquired immunogenicity of calf thymus DNA and LDL modified by D-ribose: a comparative study," International Journal of Biological Macromolecules, vol. 72, pp. 1222-1227, 2015.

[41] F. Akhter, M. S. Khan, A. A. Alatar, M. Faisal, and S. Ahmad, "Antigenic role of the adaptive immune response to d-ribose glycated LDL in diabetes, atherosclerosis and diabetes atherosclerotic patients," Life Sciences, vol. 151, pp. 139-146, 2016.

[42] F. Akhter, M. Salman Khan, M. Faisal, A. A. Alatar, and S. Ahmad, "Detection of circulating auto-antibodies against ribosylated-LDL in diabetes patients," Journal of Clinical Laboratory Analysis, vol. 31, no. 2, article e22039, 2017.

[43] Z. Siddiqui, M. Faisal, A. R. Alatar, and S. Ahmad, "Prevalence of auto-antibodies against D-ribose-glycated-hemoglobin in diabetes mellitus," Glycobiology, vol. 29, no. 5, pp. 409-418, 2019.

[44] M. Y. Khan, S. Alouffi, M. S. Khan, F. M. Husain, F. Akhter, and S. Ahmad, "The neoepitopes on methylglyoxal (MG) glycated LDL create autoimmune response; autoimmunity detection in T2DM patients with varying disease duration," Cellular Immunology, vol. 351, article 104062, 2020.

[45] S. Ahmad, Moinuddin, K. Dixit, U. Shahab, K. Alam, and A. Ali, "Genotoxicity and immunogenicity of DNA-advanced glycation end products formed by methylglyoxal and lysine in presence of $\mathrm{Cu}^{2+}$, Biochemical and Biophysical Research Communications, vol. 407, no. 3, pp. 568-574, 2011.

[46] S. Alam, Z. Arif, and K. Alam, "Glycated-H2A histone is better bound by serum anti-DNA autoantibodies in SLE patients: glycated-histones as likely trigger for SLE?," Autoimmunity, vol. 48, no. 1, pp. 19-28, 2015.

[47] U. Shahab, S. Tabrez, M. S. Khan et al., "Immunogenicity of DNA-advanced glycation end product fashioned through glyoxal and arginine in the presence of $\mathrm{Fe}^{3+}$ : its potential role in prompt recognition of diabetes mellitus auto-antibodies," Chemico-Biological Interactions, vol. 219, pp. 229-240, 2014.

[48] U. Shahab, S. A. Moinuddin, S. Ahmad et al., "Genotoxic effect of N-hydroxy-4-acetylaminobiphenyl on human DNA: implications in bladder cancer," PLoS One, vol. 8, no. 1, article e53205, 2013.

[49] R. N. Johnson, P. A. Metcalf, and J. R. Baker, "Fructosamine: a new approach to the estimation of serum glycosylprotein. An index of diabetic control," Clinica Chimica Acta, vol. 127, no. 1, pp. 87-95, 1983.

[50] N. A. Ansari, Moinuddin, A. R. Mir et al., "Role of early glycation Amadori products of lysine-rich proteins in the produc- tion of autoantibodies in diabetes type 2 patients," Cell Biochemistry and Biophysics, vol. 70, no. 2, pp. 857-865, 2014.

[51] M. P. Cohen, E. A. Shea, and V. Y. Wu, "Inhibiting low-density lipoprotein glycation ameliorates increased cholesteryl ester synthesis in macrophages and hypercholesterolemia and aortic lipid peroxidation in streptozotocin diabetic rats," Metabolism: Clinical and Experimental, vol. 59, no. 5, pp. 658-663, 2010.

[52] J. M. Ashraf, U. Shahab, S. Tabrez et al., "DNA glycation from 3-deoxyglucosone leads to the formation of AGEs: potential role in cancer auto-antibodies," Cell Biochemistry and Biophysics, vol. 74, no. 1, pp. 67-77, 2016.

[53] R. L. Levine, J. A. Williams, E. R. Stadtman, and E. Shacter, "Carbonyl assays for determination of oxidatively modified proteins," Methods in Enzymology, vol. 233, pp. 346-357, 1994.

[54] M. A. Khan, Z. Arif, M. A. Khan, Moinuddin, and K. Alam, "Methylglyoxal produces more changes in biochemical and biophysical properties of human IgG under high glucose compared to normal glucose level," PLoS One, vol. 13, no. 1, article e0191014, 2018.

[55] S. Ahmad, F. Akhter, Moinuddin, U. Shahab, and M. S. Khan, "Studies on glycation of human low density lipoprotein: A functional insight into physico-chemical analysis," International Journal of Biological Macromolecules, vol. 62, pp. 167$171,2013$.

[56] H. Ohkawa, N. Ohishi, and K. Yagi, "Assay for lipid peroxides in animal tissues by thiobarbituric acid reaction," Analytical Biochemistry, vol. 95, no. 2, pp. 351-358, 1979.

[57] M. L. Kakade and I. E. Liener, "Determination of available lysine in proteins," Analytical Biochemistry, vol. 27, no. 2, pp. 273-280, 1969.

[58] N. A. Ansari, Moinuddin, and R. Ali, "Glycated Lysine Residues: A Marker for Non-Enzymatic Protein Glycation in Age- Related Diseases," Disease Markers, vol. 30, no. 6, pp. 317-324, 2011.

[59] M. Y. Khan, S. Alouffi, and S. Ahmad, "Immunochemical studies on native and glycated LDL - An approach to uncover the structural perturbations," International Journal of Biological Macromolecules, vol. 115, pp. 287-299, 2018.

[60] H. Khan, M. S. Khan, and S. Ahmad, "The in vivo and in vitro approaches for establishing a link between advanced glycation end products and lung cancer," Journal of Cellular Biochemistry, vol. 119, no. 11, pp. 9099-9109, 2018.

[61] M. Azevedo, J. Falcao, J. Raposo, and C. Manso, "Superoxide radical generation by Amadori compounds," Free Radical Research Communications, vol. 4, no. 5, pp. 331-335, 1988.

[62] T. Sakurai and S. Tsuchiya, "Superoxide production from nonenzymatically glycated protein," FEBS Letters, vol. 236, no. 2, pp. 406-410, 1988.

[63] H. Sugiuchi, T. Irie, Y. Uji et al., "Homogeneous assay for measuring low-density lipoprotein cholesterol in serum with triblock copolymer and alpha-cyclodextrin sulfate," Clinical Chemistry, vol. 44, no. 3, pp. 522-531, 1998.

[64] G. Rabbani, E. Ahmad, N. Zaidi, S. Fatima, and R. H. Khan, "PH-induced molten globule state of Rhizopus niveus lipase is more resistant against thermal and chemical denaturation than its native state," Cell Biochemistry and Biophysics, vol. 62, no. 3, pp. 487-499, 2012.

[65] M. Abidi, M. S. Khan, S. Ahmad et al., "Biophysical and biochemical studies on glycoxidatively modified human low density lipoprotein," Archives of Biochemistry and Biophysics, vol. 645, pp. 87-99, 2018. 
[66] M. Sauer, J. Hofkens, and J. Enderlein, Handbook of Fluorescence Spectroscopy and Imaging, Handbook of Fluorescence Spectroscopy and Imaging, Wiley-VCH, 2011.

[67] J. V. Hunt, M. A. Bottoms, and M. J. Mitchinson, “Oxidative alterations in the experimental glycation model of diabetes mellitus are due to protein-glucose adduct oxidation. Some fundamental differences in proposed mechanisms of glucose oxidation and oxidant production," Biochemical Journal, vol. 291, no. 2, pp. 529-535, 1993.

[68] M. F. Beal, "Oxidatively modified proteins in aging and disease1, 2," Free Radical Biology and Medicine, vol. 32, no. 9, pp. 797-803, 2002.

[69] I. Dalle-Donne, R. Rossi, D. Giustarini, A. Milzani, and R. Colombo, "Protein carbonyl groups as biomarkers of oxidative stress," Clinica Chimica Acta, vol. 329, no. 1-2, pp. 23-38, 2003.

[70] H. Mirzaei and F. Regnier, "Enrichment of carbonylated peptides using Girard P reagent and strong cation exchange Chromatography," Analytical Chemistry, vol. 78, no. 3, pp. 770778, 2006.

[71] D. Suzuki and T. Miyata, "Carbonyl stress in the pathogenesis of diabetic nephropathy," Internal Medicine, vol. 38, no. 4, pp. 309-314, 1999.

[72] R. Jha and S. I. Rizvi, "Carbonyl formation in erythrocyte membrane proteins during aging in humans," Biomedical Papers of the Medical Faculty of the University Palacky, Olomouc, Czech Republic, vol. 155, no. 1, pp. 39-42, 2011.

[73] R. Ahmad, A. K. Tripathi, P. Tripathi, S. Singh, R. Singh, and R. K. Singh, "Malondialdehyde and protein carbonyl as biomarkers for oxidative stress and disease progression in patients with chronic myeloid leukemia," in vivo, vol. 22, no. 4, pp. 525-528, 2008.

[74] P. J. Thornalley, S. Battah, N. Ahmed et al., "Quantitative screening of advanced glycation endproducts in cellular and extracellular proteins by tandem mass spectrometry," Biochemical Journal, vol. 375, no. 3, pp. 581-592, 2003.

[75] N. Ahmed, "Advanced glycation endproducts-role in pathology of diabetic complications," Diabetes Research and Clinical Practice, vol. 67, no. 1, pp. 3-21, 2005.

[76] S. Rehman, S. Alouffi, M. Faisal, A. A. Qahtan, A. A. Alatar, and S. Ahmad, "Methylglyoxal mediated glycation leads to neo-epitopes generation in fibrinogen: role in the induction of adaptive immune response," International Journal of Biological Macromolecules, vol. 175, pp. 535-543, 2021. 